Rev. Mat. Iberoamericana 21 (2005), no. 3, 729-770

\title{
Asymptotic windings over the trefoil knot
}

Jacques Franchi

\begin{abstract}
Consider the group $G:=P S L_{2}(\mathbb{R})$ and its subgroups $\Gamma:=P S L_{2}(\mathbb{Z})$ and $\Gamma^{\prime}:=D S L_{2}(\mathbb{Z}) . G / \Gamma$ is a canonical realization (up to an homeomorphism) of the complement $\mathbb{S}^{3} \backslash T$ of the trefoil knot $T$, and $G / \Gamma^{\prime}$ is a canonical realization of the 6-fold branched cyclic cover of $\mathbb{S}^{3} \backslash T$, which has 3-dimensional cohomology of 1-forms.

Putting natural left-invariant Riemannian metrics on $G$, it makes sense to ask which is the asymptotic homology performed by the Brownian motion in $G / \Gamma^{\prime}$, describing thereby in an intrinsic way part of the asymptotic Brownian behavior in the fundamental group of the complement of the trefoil knot. A good basis of the cohomology of $G / \Gamma^{\prime}$, made of harmonic 1-forms, is calculated, and then the asymptotic Brownian behavior is obtained, by means of the joint asymptotic law of the integrals of the above basis along the Brownian paths.

Finally the geodesics of $G$ are determined, a natural class of ergodic measures for the geodesic flow is exhibited, and the asymptotic geodesic behavior in $G / \Gamma^{\prime}$ is calculated, by reduction to its Brownian analogue, though it is not precisely the same (counter to the hyperbolic case).
\end{abstract}

\section{Introduction}

Most knots obey the celebrated uniformization theorem of Thurston ([19]): their complement in $\mathbb{S}^{3}$ are homeomorphic to a unique complete hyperbolic manifold of finite volume, which assigns then a canonical geometrical structure to these manifolds. In this homeomorphism the knot is sent to infinity, namely to the unique cusp of the hyperbolic manifold.

2000 Mathematics Subject Classification: Primary: 58J65. Secondary: 60J65, 37D40, 37D30, 37A50, 20H05, 53C22.

Keywords: Trefoil knot, modular group, quasi-hyperbolic manifold, harmonic 1-forms, Brownian motion, geodesics, ergodic measures, geodesic flow, asymptotic laws. 
In this canonical hyperbolic context, it makes sense and it is possible to compute the asymptotic Brownian and geodesic behaviors. This has been done in [6]. See also $[4,2,3]$.

The trefoil knot $T$ is the simplest of non-trivial torical and then nonuniformizable knots. There is a classical algebraic realization of $T$ as a simple curve in $\mathbb{C}^{2}$, but this does not correspond to a precise canonical geometric structure, and the asymptotic Brownian windings about $T$ one can compute in such model (the quantity computed so is the length of the knot (trefoil or other) divided by the global volume, see [5]) is not intrinsic, and does not resemble anyway the uniformization.

Now, there exists however for the complement of this particular knot some intrinsic geometrical structure, replacing the non-existing hyperbolic structure. Indeed it is known $([13,9])$ that the complement of $T$ in $\mathbb{S}^{3}$ is homeomorphic to the quotient $G / \Gamma$ of the group $G:=P S L_{2}(\mathbb{R})$ by its modular subgroup $\Gamma:=P S L_{2}(\mathbb{Z})$. Moreover it happens that any left-invariant Riemannian structure on this homogeneous space $G / \Gamma$ is quasi-hyperbolic (and hyperbolic in the sense of Gromov), with a unique cusp corresponding to the trefoil $\operatorname{knot} T$. The 1-cohomology of this space is a real line, generated by the harmonic 1-form computing the linking number about $T$.

Moreover this canonical structure for the complement of $T$ admits a less known interesting feature. Among the cyclic branched covers of the complement of $T$, which exhibit a 6 -fold periodicity, one unique presents an increase of the first Betti number : the 6-fold cyclic branched cover $\Sigma_{6}^{\prime}$ of the complement of $T$ in $\mathbb{S}^{3}$ has a 3-dimensional cohomology of 1-forms. (For these questions, see [14]. For example, $\Sigma_{5}$ is the Poincaré sphere.) $\Sigma_{6}=\Sigma_{6}^{\prime} \cup T$ is a smooth compact manifold, and $H^{1}\left(\Sigma_{6}\right) \equiv \mathbb{R}^{2}$ corresponds to two "angles" accounting for more information on the fundamental group of $\mathbb{S}^{3} \backslash T$ than the linking number 1-form alone. Of course taking also this linking number into account, we have $H^{1}\left(\Sigma_{6}^{\prime}\right) \equiv \mathbb{R}^{3}$. Note that the two "angles" just mentionned are computed by bounded 1-forms, counter to the linking number 1-form.

Now it happens that this cover $\Sigma_{6}^{\prime}$ is in turn homeomorphic to $G / \Gamma^{\prime}$, $\Gamma^{\prime}:=D S L_{2}(\mathbb{Z})$ being the subgroup of the modular group $\Gamma$ generated by its commutators (see Proposition 1 below). So we have a canonical structure for this interesting cyclic cover of the complement of $T$, at least once some natural left-invariant Riemannian metric is chosen on the Lie group $G$. We fix such metrics (depending on a real parameter $a$ ) by taking orthonormal some basis of the Lie algebra $s \ell_{2}(\mathbb{R})$ which diagonalizes the Killing form and is made of symmetrical and skew-symmetrical elements, and which is simply expressed in the Iwasawa coordinates. They happen to be canonical in a natural geometrical sense, viewing $G$ as $T^{1} \mathbb{H}^{2}$, and pertain to the 6 th of the 
eight 3-dimensional geometries described by Thurston ([19]), viewing $G / \Gamma^{\prime}$ as $T^{1}\left(\mathbb{H}^{2} / \Gamma^{\prime}\right)$.

It becomes now fairly natural to compute in this canonical model the asymptotic behavior of the Brownian motion. This canonical Riemannian manifold $G / \Gamma^{\prime}$ is quasi-hyperbolic, hyperbolic in the sense of Gromov, has finite volume, one unique cusp, and projects (by annihiling the maximal compact subgroup of $G$, a circle) onto $\mathbb{H}^{2} / \Gamma^{\prime}$, a hyperbolic surface of genus 1 , which carries the two "angles" mentionned above. These "angles" give raise to regular windings and then to a central limit theorem, while the linking number will be given roughly by windings around the cusp and then will generate singular windings.

To perform the computations, of the harmonic forms (see Theorem 1 below) and of their stochastic line-integrals along the Brownian paths, and then of the asymptotic behaviors of those integrals (see Theorem 2 below), we use the Iwasawa coordinates on $G$, taking advantage of this parametrization without singularity, which moreover shows up the hyperbolic part of $G$.

To be more precise, let us mention that our harmonic forms are very conveniently expressed (in Theorem 1 below) in terms of $\eta^{4}$, the 4 th power of the classical Dedekind $\eta$ function: indeed the two handle (bounded) harmonic forms are given by $\eta^{4}(z) d z$, and the linking number (unbounded) harmonic form is given by $d \theta+d\left(\arg \eta^{4}(z)\right), \theta$ denoting in $T^{1}\left(\mathbb{H}^{2} / \Gamma^{\prime}\right)$ the parameter of the circle above $z \in \mathbb{H}^{2} / \Gamma^{\prime}$.

The asymptotic Brownian homology on $G / \Gamma^{\prime}$ is now given by the asymptotic behavior of the 3-dimensional martingale obtained by integrating along the Brownian paths (up to time $t$ ) the 3 basic harmonic forms found in Theorem 1. Theorem 2 describes this asymptotic behavior by the following convergence in law, after normalization of the fast martingale by $t$ and of the slow ones by $\sqrt{t}$ : the fast winding component goes to a Cauchy variable (with parameter $\frac{1}{2}$ ), while the two slow winding components go to normal variables (centred with variance expressed by an integral of $|\eta|^{8}$ ); the main feature being the independence of these 3 limiting variables.

From a probabilistic and technical point of view, the arguments used here were already partly scattered in [2], [4], [5], and [6], but the major difficulty in the proof of Theorem 2 below is to establish the asymptotic independence of regular and singular windings, that is to say the independence of the limiting laws of the slow and rapid windings. Such a question, relative to this type of independence, seems not to have been yet precisely addressed. See however on one hand [7], for an analogous question concerning the geodesic flow on a surface, handled totally differently, by means of a coding method, and on the other hand [20], which states an analogous result for the Brownian motion on a Riemann surface, but without proving the asymptotic independence. 
Then the geodesics of $G$ are determined, in Proposition 2 below. They happen to project on $\mathbb{H}^{2}$ according to a generic Euclidian circle or line (intersected with $\mathbb{H}^{2}$ ), these projections having constant energy. Note that the geodesic flow is non-ergodic on the tangent bundle $T^{1}\left(G / \Gamma^{\prime}\right)$.

However the description of geodesics (see Proposition 2) allows to exhibit a natural class of ergodic measures for the geodesic flow on $G / \Gamma^{\prime}$, each one carried by a leaf of $T^{1}\left(G / \Gamma^{\prime}\right)$. Fixing any such ergodic invariant probability measure, the asymptotic geodesic behavior in $G / \Gamma^{\prime}$ is calculated, by means of the joint asymptotic law of the integrals of the harmonic basis along geodesics. Indeed Theorem 3 below describes this asymptotic law: it is similar to the asymptotic law calculated in Theorem 2, but differs though by a constant depending on the parameters of the metric and of the leaf, and mainly by an additional contribution in the fast component, for which the circle form $d \theta$ is responsible.

The method for getting the geodesic result is based on a reduction to the Brownian behavior (calculated in Theorem 2), as in the series of articles $[2,3,4,6,12]$. There are however some noteworthy simplifications in comparison with the proofs in these articles, mainly due to the harmonicity of the integrated 1-forms, as in [11]. In particular, there is no more need of a spectral gap, nor to use a foliated diffusion. Another change (and hopefully clarification) with respect to these previous proofs is the use of a simultaneous disintegration of the Liouville and the Wiener measures: we avowedly condition the Brownian motion (starting from a given point $z \in \mathbb{H}^{2}$ ) to exit the hyperbolic plane at the same point as a given geodesic (starting also from $z$ ). This point of view was more or less implicit in the preceding proofs, but did not really appear.

Finally it is worth noticing again that, counter to the hyperbolic case of $[2,3,4,6,7,11,12]$, the geodesic and Brownian asymptotic behaviors are here no longer the same, though comparable. The spiral windings of the geodesics about their projections on $\mathbb{H}^{2}$ is mainly responsible for this feature. The main aim of these previous works, and in particular of [7], [4] and [2], which deal with surfaces, was already to compute the asymptotic windings law of geodesics, by means of integrals of winding forms, and, for [4] and [2], of comparison with the Brownian analogue. An important difference however with the present study is the non-hyperbolicity (in the strict sense) and non-ergodicity of the present underlying manifold $G / \Gamma^{\prime}$, which allows a different asymptotic behavior between diffusions and geodesics. Another sensible difference is that these previous works did not consider jointly fast and slow Brownian windings. Indeed, while [4] and [2] deal only with fast windings, [7] treats both sorts, but only for geodesics. 
It is a pleasure to thank J.P. Wintenberger for having drawn my attention on congruence groups, Hurwitz formula, and Shimura's book, and T. Delzant for a useful remark.

\section{The commutator subgroup of the modular group}

Consider the group $G:=P S L_{2}(\mathbb{R})$, its modular subgroup $\Gamma:=P S L_{2}(\mathbb{Z})$ and

$\Gamma^{\prime}:=D S L_{2}(\mathbb{Z})$ the subgroup of $\Gamma$ generated by its commutators.

As usual, let us identify $G$ with the unitary tangent bundle $T^{1} \mathbb{H}^{2} \equiv \mathbb{H}^{2} \times \mathbb{S}^{1}$ of the hyperbolic plane $\mathbb{H}^{2} \equiv \mathbb{R} \times \mathbb{R}_{+}^{*}$, and also with the group of Möbius isometries (homographies) of $\mathbb{H}^{2}$.

Let us distinguish the following elements $u, v, \beta, \gamma$ of $G$, defined by:

$$
\begin{array}{ll}
u(z):=-1 / z, & v(z):=(z-1) / z, \\
\beta(z):=(2 z+1) /(z+1), & \gamma(z):=(z+1) /(z+2) .
\end{array}
$$

Consider also the subgroup $\Gamma_{6}$ of elements of $\Gamma$ which are congruent to the unity modulo 6 , that is to say equivalently: $\Gamma_{6}$ is the kernel of the natural projection $p$ of $\Gamma$ onto $P S L_{2}(\mathbb{Z} / 6 \mathbb{Z})$. Let $\left[\alpha_{1}, \alpha_{2}\right]:=\alpha_{1} \alpha_{2} \alpha_{1}^{-1} \alpha_{2}^{-1}$ denote as usual the commutator of $\alpha_{1}, \alpha_{2} \in G$.

Lemma 1 The group $\Gamma$ is generated by $\{u, v\}$ and admits the presentation $\left\{u, v \mid u^{2}=v^{3}=1\right\}$; The group $\Gamma^{\prime}$ is the free group generated by $\{\beta, \gamma\}$; The quotient group $\Gamma / \Gamma^{\prime}$ is isomorphic to $\mathbb{Z} / 6 \mathbb{Z}$. Moreover we have: $\beta=[v, u], \gamma=\left[v^{-1}, u\right], v u(z)=z+1,\left[\beta, \gamma^{-1}\right]=(v u)^{6}=(z \mapsto z+6) \in \Gamma_{6}$.

Denote by $D_{0}$ the most usual fundamental domain for the action of the modular group $\Gamma$ on the hyperbolic Poincaré plane $\mathbb{H}^{2}: D_{0}$ is the ideal triangle of $\mathbb{H}^{2}$ delimited by the vertical half-lines $\left[e^{\sqrt{-1} 2 \pi / 3}, \infty\right]$ and $\left[e^{\sqrt{-1} \pi / 3}, \infty\right]$ and the segment of the trigonometric circle delimited by $e^{\sqrt{-1} \pi / 3}, e^{\sqrt{-1} 2 \pi / 3}$. Recall that $\{1, u\}$ is the stabilisator of the elliptic point $\sqrt{-1}$, and $\left\{1, v, v^{2}\right\}$ is the stabilisator of the elliptic point $e^{\sqrt{-1} \pi / 3} \equiv e^{\sqrt{-1} 2 \pi / 3}$.

Then we have the following fundamental domains for the action of $\Gamma^{\prime}$ :

$$
D_{0} \cup v D_{0} \cup v^{2} D_{0} \cup u D_{0} \cup v u D_{0} \cup v^{2} u D_{0},
$$

and

$$
D:=D_{0} \cup \beta^{-1} v \beta^{-1} D_{0} \cup \beta v^{2} \beta D_{0} \cup \beta u \gamma D_{0} \cup v u D_{0} \cup v^{2} u \beta^{-1} D_{0} .
$$

As $\beta^{-1} v \beta^{-1}(z)=z-2, \beta v^{2} \beta(z)=z+2, \beta u \gamma(z)=z+3, v^{2} u \beta^{-1}(z)=z-1$, we see that $D$ is merely the union of the translates of $D_{0}$ successively by $-2,-1,0,1,2,3$. 
We have the following identifications on the boundary of $D$ :

$$
\begin{aligned}
& \gamma\left(e^{\sqrt{-1} 2 \pi / 3}-2, \sqrt{-1}-2, e^{\sqrt{-1} 2 \pi / 3}-1\right)=\left(e^{\sqrt{-1} 2 \pi / 3}+2, \sqrt{-1}+1, e^{\sqrt{-1} 2 \pi / 3}+1\right), \\
& \beta\left(e^{\sqrt{-1} 2 \pi / 3}-1, \sqrt{-1}-1, e^{\sqrt{-1} 2 \pi / 3}\right)=\left(e^{\sqrt{-1} 2 \pi / 3}+3, \sqrt{-1}+2, e^{\sqrt{-1} 2 \pi / 3}+2\right), \\
& \beta \gamma^{-1}\left(e^{\sqrt{-1} 2 \pi / 3}, \sqrt{-1}, e^{\sqrt{-1} 2 \pi / 3}+1\right)=\left(e^{\sqrt{-1} 2 \pi / 3}+4, \sqrt{-1}+3, e^{\sqrt{-1} 2 \pi / 3}+3\right), \\
& {\left[\beta, \gamma^{-1}\right]\left(e^{\sqrt{-1} 2 \pi / 3}-2+\sqrt{-1} \mathbb{R}_{+}\right)=e^{\sqrt{-1} 2 \pi / 3}+4+\sqrt{-1} \mathbb{R}_{+} .}
\end{aligned}
$$

Lemma $2 \quad \Gamma^{\prime}$ is a congruence group. More precisely, we have $\Gamma_{6} \subset \Gamma^{\prime}$, and in $\Gamma^{\prime}$ a word in $\{\beta, \gamma\}$ belongs to $\Gamma_{6}$ if and only if its total weights with respect to $\beta$ and $\gamma$ are even and equal modulo 6 . Moreover $\left[\Gamma^{\prime}: \Gamma_{6}\right]=12=$ $\operatorname{Card}\left(p\left(\Gamma^{\prime}\right)\right)$, the quotient group $p(\Gamma) / p\left(\Gamma^{\prime}\right)$ is isomorphic to $\mathbb{Z} / 6 \mathbb{Z}$, and the quotient group $\Gamma^{\prime} / \Gamma_{6}$ is isomorphic to $\mathbb{Z} / 6 \mathbb{Z} \times \mathbb{Z} / 2 \mathbb{Z}$.

Proof. We saw with the fundamental domain $D$ above that $\Gamma / \Gamma^{\prime}$ is made of the cosets $(z \mapsto z+k) \Gamma^{\prime}$, for $k \in\{0, \ldots, 5\}$. Then we have $\left[p(\beta), p(\gamma)^{-1}\right]=$ $p\left(\left[\beta, \gamma^{-1}\right]\right)=1$, showing that $p(\beta)$ and $p(\gamma)$ commute. Thus for any integers $m, n$ the element $p\left(\beta^{m} \gamma^{n}\right)$ is (as a matrix) symmetrical in $p(\Gamma)$, that is to say symmetrical or skew-symmetrical as a matrix in $S L_{2}(\mathbb{Z} / 6 \mathbb{Z})$, since $\beta$ and $\gamma$ are.

Moreover, $p(\beta)^{6}=p(\gamma)^{6}=1$, and

$$
p(\Gamma)=\left\{p\left((z \mapsto z+k) \beta^{m} \gamma^{n}\right) \mid 0 \leq k \leq 5,0 \leq m, n \leq 5\right\} .
$$

And $p\left((z \mapsto z+k) \beta^{m} \gamma^{n}\right)=1 \Longleftrightarrow\left(\begin{array}{cc}a+k \varepsilon b & b+k d \\ b \varepsilon & d\end{array}\right)=\left(\begin{array}{cc}\varepsilon^{\prime} & 0 \\ 0 & \varepsilon^{\prime}\end{array}\right)$ modulo 6, where $\varepsilon, \varepsilon^{\prime}= \pm 1$ and $p\left(\beta^{m} \gamma^{n}\right)=\left(\begin{array}{cc}a & b \\ b \varepsilon & d\end{array}\right)$, showing that

$$
p\left((z \mapsto z+k) \beta^{m} \gamma^{n}\right)=1 \Longleftrightarrow k=0 \text { and } p(\beta)^{m} p(\gamma)^{n}=1
$$

Hence we have shown that $\Gamma_{6} \subset \Gamma^{\prime}$, and also the second statement of the lemma, since it is easily seen that

$$
p(\beta)^{m} p(\gamma)^{n}=1 \Longleftrightarrow m \in 2 \mathbb{Z} \text { and } m-n \in 6 \mathbb{Z} \text {. }
$$

The above also implies the isomorphism between $p(\Gamma) / p\left(\Gamma^{\prime}\right)$ and $\mathbb{Z} / 6 \mathbb{Z}$, and that the kernel of $\left(\mathbb{Z} / 6 \mathbb{Z} \ni(m, n) \longmapsto p(\beta)^{m} p(\gamma)^{n} \in p\left(\Gamma^{\prime}\right)\right)$ is isomorphic to $\mathbb{Z} / 3 \mathbb{Z}$. Whence $\operatorname{Card}\left(p\left(\Gamma^{\prime}\right)\right)=12,\left[\Gamma: \Gamma_{6}\right]=\operatorname{Card}(p(\Gamma))=72$, and $\left[\Gamma^{\prime}: \Gamma_{6}\right]=72 / 6=12$. The last claim is now clear from the above, which shows that $\Gamma^{\prime} / \Gamma_{6}$ is generated by the cosets of $\beta$ and $\beta \gamma$, which commute and are of order respectively 6 and 2 . 


\section{Modular forms on $\mathbb{H}^{2} / \Gamma^{\prime}$}

The Hurwitz formula (see for example [17, Section 1.5]) asserts in the present case that

$$
2 \times \operatorname{genus}\left(\mathbb{H}^{2} / \Gamma^{\prime}\right)-2=6 \times\left(2 \times \operatorname{genus}\left(\mathbb{H}^{2} / \Gamma\right)-2\right)+\sum_{z \in \mathbb{H}^{2} / \Gamma^{\prime}}(r(z)-1)
$$

where $r(z)$ denotes the ramification index of the covering $\left(\mathbb{H}^{2} / \Gamma^{\prime} \rightarrow \mathbb{H}^{2} / \Gamma\right)$ at $z$, which satisfies

$$
\sum_{z \text { above } z^{\prime}} r(z)=6 \quad \text { for any } z^{\prime} \in \mathbb{H}^{2} / \Gamma \text {. }
$$

Here the ramifications occur only above $\infty, \sqrt{-1}$, and $e^{\sqrt{-1} 2 \pi / 3}$. There are one point above $\infty$, with index 6 , three points above $\sqrt{-1}$, with index 2 , and two points above $e^{\sqrt{-1} 2 \pi / 3}$, with index 3 . Since $\mathbb{H}^{2} / \Gamma$ is homeomorphic to $\mathbb{H}^{2}$, and then has genus 0 , we get the genus 1 for $\mathbb{H}^{2} / \Gamma^{\prime}$.

Now, theorems 2.23 and 2.24 of ([17], Section 2.6, case $k=2$ and genus $=1$ ) assert that the modular forms $f(z) d z$, for $\mathbb{H}^{2} / \Gamma^{\prime}$ as for $\mathbb{H}^{2} / \Gamma_{6}$, are all proportional.

We need next the $\eta$ function of Dedekind, defined on $\mathbb{H}^{2}$ (seen as the Poincaré half-plane) by:

$$
\eta(z):=e^{\sqrt{-1} \pi z / 12} \times \prod_{n \in \mathbb{N}^{*}}\left(1-e^{\sqrt{-1} 2 \pi n z}\right) .
$$

We shall also need its logarithmic derivative:

$$
\tilde{\eta}(z):=\eta^{\prime}(z) / \eta(z)=\sqrt{-1} \frac{\pi}{12}-2 \pi \sqrt{-1} \sum_{n \in \mathbb{N}^{*}} \frac{n}{e^{-\sqrt{-1} 2 \pi n z}-1},
$$

which converges absolutely uniformly in $D . \eta$ and $\tilde{\eta}$ are holomorphic, and bounded in $D$.

$\eta$ clearly satisfies $\eta(z+1)=e^{\sqrt{-1} \pi / 12} \eta(z)$, and is known (see for example [1, Theorem 3.1 of Section III.3]) to satisfy also (with as the square root the usual principal branch):

$$
\eta(-1 / z)=e^{-\sqrt{-1} \pi / 4} \sqrt{z} \eta(z) .
$$

As a consequence, $\eta(g(z))=\eta(z) \times g^{\prime}(z)^{-1 / 4}$ up to some 24 -fold root of 1 (depending on $g$ ) for any $g \in \Gamma$, and in particular $\eta(g(z))=\eta(z) \times g^{\prime}(z)^{-1 / 4}$ for any $g \in \Gamma^{\prime}$. 
Hence we have the first sentence of the following lemma (owing to the canonical injection of $H^{1}\left(\mathbb{H}^{2} / \Gamma^{\prime}\right)$ into $H^{1}\left(G / \Gamma^{\prime}\right)$, induced by the canonical projection $g \mapsto g(\sqrt{-1})$ from $G$ onto $\left.\mathbb{H}^{2}\right)$. The second sentence is obtained merely by differentiating the above relation for $\eta$. The third is straightforward from the above expressions of $\eta$ and $\tilde{\eta}$.

\section{Lemma 3}

(i) $\eta^{4}(z) d z$ is a closed modular form on $\mathbb{H}^{2} / \Gamma^{\prime}$, and thus on $G / \Gamma^{\prime}$.

(ii) We have $\tilde{\eta}(-1 / z)=z^{2} \times \tilde{\eta}(z)+z / 2$ and $\tilde{\eta}(z+1)=\tilde{\eta}(z)$ for any $z \in \mathbb{H}^{2}$.

(iii) We have in $D: \eta(z)=\mathcal{O}\left(e^{-\pi y / 12}\right)$ and $\tilde{\eta}(z)=\sqrt{-1} \pi / 12+\mathcal{O}\left(e^{-2 \pi y}\right)$.

Remark 1 Set

$$
\Theta(z):=\sum_{n \in \mathbb{Z}} e^{\sqrt{-1} \pi n^{2} z},
$$

which is holomorphic in $\mathbb{H}^{2}$. Poisson formula applied to the function $n \mapsto$ $e^{-\pi y n^{2}}$ shows that (for $z=\sqrt{-1} y$ and then for any $z \in \mathbb{H}^{2}$ )

$$
\Theta(-1 / z)=e^{-\sqrt{-1} \pi / 4} \sqrt{z} \Theta(z)
$$

as for $\eta$ above. But this theta function $\Theta$ is only 2-periodic, and has no 1-periodic power, so that (since $v u=\beta u \gamma(v u)^{-2}$ ) it cannot be used to get a $\Gamma^{\prime}$-automorphic form.

\section{Link with the trefoil knot}

Denote by $T$ the trefoil knot, realized in the sphere $\mathbb{S}^{3}$. Denote by $\Sigma_{k}$ the $k$-fold cyclic branched cover of its complement $\mathbb{S}^{3} \backslash T$, for $k \in \mathbb{N}^{*}$. See [14], in particular $([14, \mathrm{X}, \mathrm{C}, \mathrm{D}],[14, \mathrm{VI}, \mathrm{B}]$ and $[14, \mathrm{VII}, \mathrm{D}]) . \Sigma_{k}$ is a compact 3dimensional smooth manifold, $H_{1}\left(\Sigma_{k}\right)=H_{1}\left(\Sigma_{k+6}\right)$, and $H^{1}\left(\Sigma_{k}\right)$ is trivial for $k \notin 6 \mathbb{N}$. (A pleasant feature, irrelevant here, is that $\Sigma_{5}$ is the Poincaré 3-sphere.)

It is not very hard to see that $H_{1}\left(\Sigma_{6}\right) \equiv \mathbb{Z}^{2}$. Indeed $\Pi_{1}\left(\Sigma_{6} \backslash T\right)$ is made of those lifts of elements in $\Pi_{1}\left(\mathbb{S}^{3} \backslash T\right)$ which are still loops, that is those which have linking number (with respect to $T$ ) belonging to $6 \mathbb{Z}$. This implies that $\Pi_{1}\left(\Sigma_{6} \backslash T\right.$ ) (up to an isomorphism) is generated by the center (isomorphic to $\mathbb{Z}$ ) and the commutator subgroup (free on two generators) of $\Pi_{1}\left(\mathbb{S}^{3} \backslash T\right)$. Whence the abelianised $H_{1}\left(\Sigma_{6} \backslash T\right) \equiv \mathbb{Z}^{3}$, the $H_{1}\left(\Sigma_{6}\right) \equiv \mathbb{Z}^{2}$ component corresponding to the commutator subgroup contribution.

The real cohomology of 1 -forms of $\mathbb{S}^{3} \backslash T, H^{1}\left(\mathbb{S}^{3} \backslash T\right)$, identified with the space of harmonic 1-forms on $\mathbb{S}^{3} \backslash T$, is generated by the harmonic 1-form 
computing the linking number about $T$. Considering $\Sigma_{6}^{\prime}:=\Sigma_{6} \backslash T$, we have a 6-fold cover of $\mathbb{S}^{3} \backslash T$ which has a three-dimensional real cohomology of 1 forms: $H^{1}\left(\Sigma_{6}^{\prime}\right) \equiv \mathbb{R}^{3}$, generated by the lifts of the harmonic forms belonging to $H^{1}\left(\mathbb{S}^{3} \backslash T\right) \equiv \mathbb{R}$ and the restrictions of the harmonic forms belonging to $H^{1}\left(\Sigma_{6}\right) \equiv \mathbb{R}^{2}$.

It is convenient to think of those two more directions brought by $H^{1}\left(\Sigma_{6}\right)$ into $H^{1}\left(\Sigma_{6}^{\prime}\right)$ as two "angles" accounting for more of the complicated fundamental group of $\mathbb{S}^{3} \backslash T$, than the mere linking number 1-form generating $H^{1}\left(\mathbb{S}^{3} \backslash T\right)$ does alone.

The following proposition links this interesting cover with the preceding section, confering thereby to $\Sigma_{6}^{\prime}$ a somehow canonical structure of homogeneous space.

Proposition $1 \mathbb{S}^{3} \backslash T$ is homeomorphic to $G / \Gamma$, and moreover $\Sigma_{6}^{\prime}$ is homeomorphic to $G / \Gamma^{\prime}$.

Proof. The first assertion seems to be due to Milnor. See [13], and [9]. The second one does not seem to be widely known, but M. Boileau, F. Bonahon and T. Delzant knew it.

$G / \Gamma^{\prime}$ is clearly a cover of $G / \Gamma$, with as a fibre the cyclic group $\Gamma / \Gamma^{\prime} \equiv$ $\mathbb{Z} / 6 \mathbb{Z}$, as it must be. So it remains to be sure that this cover is indeed a cyclic branched cover, and namely that near the trefoil knot the canonical projection is diffeomorphic to $\left((s, z) \mapsto\left(s, z^{6}\right)\right)$. Now, [9] shows that in $G / \Gamma$, $T$ has been sent to infinity (it corresponds to the subgroups of $\mathbb{R}^{2}$ isomorphic to $\mathbb{R}$, while its complement correspond to the lattices with unit area). And near infinity, $G / \Gamma^{\prime}$ is locally homeomorphic to the unit tangent bundle of a neighborhood of $\infty$ in the fundamental domain $D$ (with identification of its two vertical edges by means of $(z \mapsto z+6))$. It should now be clear that the cover $G / \Gamma^{\prime} \rightarrow G / \Gamma$ has the required structure near infinity, being given by

$$
((\theta, x+\sqrt{-1} y) \mapsto(\theta, 6 x+\sqrt{-1} y)),
$$

$\theta$ denoting the parameter of the circle above the point $x+\sqrt{-1} y \in D$.

Observe that the harmonic 1-forms of $G / \Gamma^{\prime} \equiv \Sigma_{6}^{\prime}$ are sums of forms of two different types: the forms arising from $G / \Gamma \equiv \mathbb{S}^{3} \backslash T$ and then from the linking number (with respect to $T$ ), which are unbounded, and the forms coming from $\Sigma_{6}$ or equivalently from the modular surface $\mathbb{H}^{2} / \Gamma^{\prime}$ (onto which $G / \Gamma^{\prime} \equiv T^{1}\left(\mathbb{H}^{2} / \Gamma^{\prime}\right)$ canonically projects, and which has 1 handle, see section 3$)$, which are bounded. 


\section{Iwasawa coordinates and metrics on $G$}

$G=P S L_{2}(\mathbb{R})$ is classically parametrized by the Iwasawa coordinates

$$
(z=x+\sqrt{-1} y, \theta) \in \mathbb{H}^{2} \times(\mathbb{R} / 2 \pi \mathbb{Z}),
$$

in the following way: each $g \in G$ writes uniquely $g=g(z, \theta):=n(x) a(y) k(\theta)$, where $n(x), a(y), k(\theta)$ are the one-parameter subgroups defined by:

$$
n(x):=\left(\begin{array}{ll}
1 & x \\
0 & 1
\end{array}\right), a(y):=\left(\begin{array}{cc}
\sqrt{y} & 0 \\
0 & 1 / \sqrt{y}
\end{array}\right), k(\theta):=\left(\begin{array}{cc}
\cos \frac{\theta}{2} & \sin \frac{\theta}{2} \\
-\sin \frac{\theta}{2} & \cos \frac{\theta}{2}
\end{array}\right),
$$

and generated respectively by the following elements of the Lie algebra $s \ell_{2}(\mathbb{R})$ :

$$
\nu:=\left(\begin{array}{ll}
0 & 1 \\
0 & 0
\end{array}\right), \alpha:=\left(\begin{array}{cc}
1 / 2 & 0 \\
0 & -1 / 2
\end{array}\right), \kappa:=\left(\begin{array}{cc}
0 & 1 / 2 \\
-1 / 2 & 0
\end{array}\right) .
$$

Note that $g=g(z, \theta) \Longleftrightarrow\left[g(\sqrt{-1})=z \quad\right.$ and $\left.\quad g^{\prime}(\sqrt{-1})=y e^{\sqrt{-1} \theta}\right]$.

Set also $\lambda:=\nu-\kappa=\left(\begin{array}{cc}0 & 1 / 2 \\ 1 / 2 & 0\end{array}\right)$, which is natural, since $\alpha, \lambda$ are symmetrical while $\kappa$ is skew-symmetrical, and since in the basis $(\alpha, \lambda, \kappa)$ of $s \ell_{2}(\mathbb{R})$ the Killing form is diagonal: it has matrix $\left(\begin{array}{ccc}-2 & 0 & 0 \\ 0 & -2 & 0 \\ 0 & 0 & 2\end{array}\right)$.

For this reason, we take on $s \ell_{2}(\mathbb{R})$ the inner product such that the basis $(\alpha, \lambda, a \kappa)$ is orthonormal, for some arbitrary parameter $a \in \mathbb{R}^{*}$. And since we want to work on the homogeneous space $G / \Gamma^{\prime}$, the Riemannian metric to be considered on $G$ must be a least $\Gamma^{\prime}$-left-invariant, and then a natural choice for the Riemannian metric on $G$ is the left-invariant metric, say $\left(\left(g_{i j}^{a}\right)\right)$, generated by the above inner product on $s \ell_{2}(\mathbb{R})$.

The simple lemma below shows that this choice of metric(s) is geometrically canonical (up to a trivial multiplicative constant), $G$ being seen as $T^{1} \mathbb{H}^{2}$. This equips $G / \Gamma^{\prime} \equiv T^{1}\left(\mathbb{H}^{2} / \Gamma^{\prime}\right)$ with the 6 th of the eight 3 dimensional geometries described by Thurston ([19]).

Let us denote by $\mathcal{L}_{\nu}, \mathcal{L}_{\alpha}, \mathcal{L}_{\kappa}, \mathcal{L}_{\lambda}$ the left-invariant vector fields on $G$ generated respectively by $\nu, \alpha, \kappa, \lambda$. A standard computation shows that

$$
\begin{aligned}
& \mathcal{L}_{\lambda}=y \sin \theta \frac{\partial}{\partial y}+y \cos \theta \frac{\partial}{\partial x}-\cos \theta \frac{\partial}{\partial \theta}, \\
& \mathcal{L}_{\alpha}=y \cos \theta \frac{\partial}{\partial y}-y \sin \theta \frac{\partial}{\partial x}+\sin \theta \frac{\partial}{\partial \theta}, \\
& \mathcal{L}_{\kappa}=\frac{\partial}{\partial \theta} .
\end{aligned}
$$


Lemma 4 The Riemannian metrics $\left(\left(g_{i j}^{a}\right)\right)$ defined above are, up to a multiplicative constant, the only ones on $G$ which are left-invariant and also invariant with respect to the action of the (Cartan compact subgroup) circle $\{k(\theta)\}$. They are given in Iwasawa coordinates $(y, x, \theta)$ by

$$
\left(\left(g_{i j}^{a}\right)\right):=\left(\begin{array}{ccc}
y^{-2} & 0 & 0 \\
0 & \left(1+a^{-2}\right) y^{-2} & a^{-2} y^{-1} \\
0 & a^{-2} y^{-1} & a^{-2}
\end{array}\right) .
$$

Proof. The left-invariant metrics on $G$ are those which are given by a constant matrix $\left(\left(a_{i j}\right)\right)$ in the basis $\mathcal{L}:=\left(\mathcal{L}_{\alpha}, \mathcal{L}_{\lambda}, \mathcal{L}_{\kappa}\right)$. Set $\mathcal{I}:=\left(\frac{\partial}{\partial y}, \frac{\partial}{\partial x}, \frac{\partial}{\partial \theta}\right)$. We have $\mathcal{I}=\mathcal{L} A$, with

$$
A:=\left(\begin{array}{ccc}
y^{-1} \cos \theta & -y^{-1} \sin \theta & 0 \\
y^{-1} \sin \theta & y^{-1} \cos \theta & 0 \\
0 & y^{-1} & 1
\end{array}\right),
$$

so that the left-invariant metrics are given in the basis $\mathcal{I}$ by ${ }^{t} A\left(\left(a_{i j}\right)\right) A$. Among them, the ones we want have to satisfy $\frac{\partial}{\partial \theta}^{t} A\left(\left(a_{i j}\right)\right) A=0$.

A direct computation shows that this is equivalent to

$$
\left(\left(a_{i j}\right)\right)=c^{2}\left(\begin{array}{ccc}
1 & 0 & 0 \\
0 & 1 & 0 \\
0 & 0 & a^{-2}
\end{array}\right),
$$

and then to $\left(\left(g_{i j}^{a}\right)\right)$ being as in the lemma.

Note that with these metrics the holomorphic form $\eta^{4}(z) d z$ of Lemma $\left.3, i\right)$ is coclosed and then harmonic.

The left Laplacian on $G$ corresponding to the basis $(\alpha, \lambda, a \kappa)$ is the Beltrami Laplacian associated with the metric $\left(\left(g_{i j}^{a}\right)\right)$, and is given by

$$
\Delta^{a}:=\mathcal{L}_{\lambda}^{2}+\mathcal{L}_{\alpha}^{2}+a^{2} \mathcal{L}_{\kappa}^{2}=y^{2}\left(\frac{\partial^{2}}{\partial y^{2}}+\frac{\partial^{2}}{\partial x^{2}}\right)-2 y \frac{\partial^{2}}{\partial \theta \partial x}+\left(1+a^{2}\right) \frac{\partial^{2}}{\partial \theta^{2}} .
$$

Note that $\mathcal{L}_{\lambda}$ and $\mathcal{L}_{\alpha}$ generate the canonical horizontal left-invariant vector fields lifted from $\mathbb{H}^{2}$ to $G, \mathbb{H}^{2}$ being endowed with its Levi-Civita connexion, so that $\Delta^{0}$ is the Bochner horizontal left Laplacian, and $\Delta^{a}=$ $\Delta^{0}+a^{2} \frac{\partial^{2}}{\partial \theta^{2}}$.

The measure

$$
\mu(d g):=\frac{d x d y d \theta}{4 \pi^{2} y^{2}}
$$

is bi-invariant, and its projection onto $G / \Gamma^{\prime}$ is a probability measure (as is easily seen by integrating over $D \times[0,2 \pi])$, proportional to the volume measure of $G / \Gamma^{\prime}$. Thus the volume of $G / \Gamma^{\prime}$ is $4 \pi^{2} /|a|$. 
Let $\tilde{y}$ denote the height in the cusp of the projection $\mathbb{H}^{2} / \Gamma^{\prime}$ of $G / \Gamma^{\prime}$, that is to say $\tilde{y}=y$ when we identify $\mathbb{H}^{2} / \Gamma^{\prime}$ with its fundamental domain $D$ (see section 2). Let us consider $\tilde{y}$ as a function on $G / \Gamma^{\prime}$ or on $\mathbb{H}^{2} / \Gamma^{\prime}$ as well, and then also as a $\Gamma^{\prime}$-invariant function on $G$ or on $\mathbb{H}^{2}$.

\section{Winding form on $G / \Gamma$}

Let us look here for the harmonic 1 -form on $G / \Gamma$, which calculates the linking number about the trefoil knot sent to infinity. Let us denote it by

$\omega_{0}=A(z, \theta) d x+B(z, \theta) d y+C(z, \theta) d \theta=U(z, \theta) d z+\bar{U}(z, \theta) d \bar{z}+C(z, \theta) d \theta$, with $A, B, C$ real and $2 U=A-\sqrt{-1} B . \quad C$ must be non-null, since $H^{1}\left(\mathbb{H}^{2} / \Gamma\right) \equiv 0$.

$\omega_{0}$ must be left-invariant under $\Gamma$, which amounts to say such that $\gamma^{*} \omega_{0}=\omega_{0}$ for $\gamma(z)=z+1$ and for $\gamma(z)=-1 / z$.

Clearly the invariance with respect to $z \mapsto z+1$ is equivalent to the 1-periodicity of $A, B, C$ (with respect to the variable $z$ ). We have then to write down the invariance with respect to $u=(z \mapsto-1 / z)$.

Now $u g(z, \theta)=g(-1 / z, \theta-2 \arg z)$, using the notation of section 5 , so that

$$
u^{*} \omega_{0}=U \circ u \frac{d z}{z^{2}}+\bar{U} \circ u \frac{d \bar{z}}{\bar{z}^{2}}+C \circ u \times\left(d \theta+\sqrt{-1}|z|^{-2}(\bar{z} d z-z d \bar{z})\right)
$$

equals $\omega_{0}$ if and only if $C$ is $\Gamma$-invariant and

$$
U(-1 / z, \theta-2 \arg z)=z^{2} U(z, \theta)-\sqrt{-1} z C(z, \theta) \quad \text { for all } z, \theta .
$$

Observe that with the metric $\left(\left(g_{i j}^{a}\right)\right)$ given in section 5 , the divergence of $\omega_{0}$ is

$$
\begin{aligned}
\delta \omega_{0} & =y^{2}\left(\frac{\partial B}{\partial y}+\frac{\partial(A-C / y)}{\partial x}+\frac{\partial\left(\frac{1+a^{2}}{y^{2}} C-A / y\right)}{\partial \theta}\right) \\
& =y^{2}\left(\frac{\partial A}{\partial x}+\frac{\partial B}{\partial y}\right)-y \frac{\partial C}{\partial x}+\frac{\partial\left(\left(1+a^{2}\right) C-y A\right)}{\partial \theta} .
\end{aligned}
$$

Hence $\omega_{0}$ is harmonic if and only if

$$
\begin{gathered}
\frac{\partial U}{\partial \bar{z}} \text { is real }, \frac{\partial U}{\partial \theta}=\frac{\partial C}{\partial z}, \\
\text { and } \frac{\partial A}{\partial x}+\frac{\partial B}{\partial y}-y^{-1} \frac{\partial C}{\partial x}+y^{-2} \frac{\partial\left(\left(1+a^{2}\right) C-y A\right)}{\partial \theta}=0
\end{gathered}
$$

which is equivalent to

$$
\frac{\partial U}{\partial \theta}=\frac{\partial C}{\partial z} \quad \text { and } \quad \frac{\partial U}{\partial \bar{z}}=4 y^{-1} \frac{\partial C}{\partial x}-4 y^{-2} \frac{\partial\left(\left(1+a^{2}\right) C-y A\right)}{\partial \theta} .
$$


Now Lemma $(3, i i)$ shows that $C \equiv 1$ and $U(z, \theta) \equiv-2 \sqrt{-1} \tilde{\eta}(z)$ is a solution.

So our 1-form $\omega_{0}$ must be

$$
\omega_{0}=d \theta+4 \mathcal{I} m(\tilde{\eta}(z)) d x+4 \mathcal{R} e(\tilde{\eta}(z)) d y,
$$

up to a multiplicative constant (which we take equal to 1 , to have the linking number calculated by $\omega_{0} / 2 \pi$, see the comment below).

Gathering this, Lemma $(3, i)$, and section 4 , we get the following:

Theorem 1 A basis of $H^{1}\left(G / \Gamma^{\prime}\right)$ is made of the 3 following harmonic 1-forms, expressed in the Iwasawa coordinates:

$$
\begin{aligned}
& \omega_{0}:=d \theta+4 \mathcal{I} m(\tilde{\eta}(z)) d x+4 \mathcal{R} e(\tilde{\eta}(z)) d y=d(\theta+4 \arg (\eta(z))), \\
& \omega_{1}:=\mathcal{R} e\left(\eta^{4}(z)\right) d x-\mathcal{I} m\left(\eta^{4}(z)\right) d y \\
& \omega_{2}:=\mathcal{I} m\left(\eta^{4}(z)\right) d x+\mathcal{R} e\left(\eta^{4}(z)\right) d y
\end{aligned}
$$

$\omega_{1}$ and $\omega_{2}$ are bounded, and $\omega_{0}=\frac{\pi}{3} d x+d \theta+\mathcal{O}\left(y e^{-2 \pi y}\right)$ near $y=\infty$.

As usual, we identify the forms on $G / \Gamma^{\prime}$ with the $\Gamma^{\prime}$-(left-)invariant forms on $G$.

Note that the norms of the forms $d x$ and $d y$ are of magnitude $\tilde{y}$ (defined in section 5), though the norm of $d \theta$ is of magnitude 1 . Then $\omega_{0}$ is unbounded, having a singularity at the cusp of $G / \Gamma^{\prime}$, and does not belong to $L^{1}\left(G / \Gamma^{\prime}, \mu\right)$.

This agrees with the different natures of the windings involved: whereas $\omega_{0}$ calculates the singular windings about the trefoil knot (even if located at infinity), $\omega_{1}$ and $\omega_{2}$ calculate regular windings around a handle, and belong to $L^{2}\left(G / \Gamma^{\prime}, \mu\right)$.

Observe that $\omega_{0}$ also calculates a sort of angle, about the trefoil knot at infinity, and that the corresponding linking number is then calculated by $\omega_{0} / 2 \pi$. This is coherent with the approximation in Theorem 1 above:

$$
\frac{\omega_{0}}{2 \pi}=\frac{d x}{6}+\mathcal{O}(1) \quad \text { near } y=\infty
$$

showing that our linking number is mainly made of the number of windings around the cusp performed by the projection on $\mathbb{H}^{2} / \Gamma^{\prime}$; note that it is clear on the fundamental domain $D$ that a loop around the cusp corresponds to an increase of $x$ by \pm 6 .

The norms mentioned above are relative to any left-invariant Riemannian structure on the Lie group $G$ (and on $G / \Gamma^{\prime}$ ). But more precisely, with respect to the precise canonical Riemannian structure $\left(\left(g_{i j}^{a}\right)\right)$ (see section 5), we have the following. 
Corollary 1 Expressed in the dual basis $\left(\mathcal{L}_{\alpha}^{*}, \mathcal{L}_{\kappa}^{*}, \mathcal{L}_{\lambda}^{*}\right)$ of $\left(\mathcal{L}_{\alpha}, \mathcal{L}_{\kappa}, \mathcal{L}_{\lambda}\right)$, we have

$$
\begin{gathered}
\omega_{0}=\mathcal{L}_{\kappa}^{*}+(\sin \theta+4 y[\operatorname{Re}(\tilde{\eta}(z)) \cos \theta-\mathcal{I} m(\tilde{\eta}(z)) \sin \theta]) \mathcal{L}_{\alpha}^{*} \\
+(-\cos \theta+4 y[\mathcal{R} e(\tilde{\eta}(z)) \sin \theta+\mathcal{I} m(\tilde{\eta}(z)) \cos \theta]) \mathcal{L}_{\lambda}^{*} \\
\omega_{1}=\left[\mathcal{R} e\left(\eta^{4}(z)\right) \cos \theta-\mathcal{I} m\left(\eta^{4}(z)\right) \sin \theta\right] y \mathcal{L}_{\lambda}^{*} \\
-\left[\operatorname{Re}\left(\eta^{4}(z)\right) \sin \theta+\mathcal{I} m\left(\eta^{4}(z)\right) \cos \theta\right] y \mathcal{L}_{\alpha}^{*} \\
\omega_{2}=\left[\operatorname{Re} e\left(\eta^{4}(z)\right) \sin \theta+\mathcal{I} m\left(\eta^{4}(z)\right) \cos \theta\right] y \mathcal{L}_{\lambda}^{*} \\
+\left[\operatorname{Re}\left(\eta^{4}(z)\right) \cos \theta-\mathcal{I} m\left(\eta^{4}(z)\right) \sin \theta\right] y \mathcal{L}_{\alpha}^{*} .
\end{gathered}
$$

As a consequence, we have

$$
\begin{aligned}
\left|\omega_{0}\right|^{2} & =1+a^{2}+16 y^{2}|\tilde{\eta}(z)|^{2}-8 y \mathcal{I} m(\tilde{\eta}(z)), \\
\left|\omega_{1}\right|^{2} & =\left|\omega_{2}\right|^{2}=y^{2}|\eta(z)|^{8}, \quad\left\langle\omega_{1}, \omega_{2}\right\rangle=0, \\
\left\langle\omega_{0}, \omega_{1}\right\rangle & =4 y^{2}\left[\mathcal{R} e\left(\eta^{4}(z)\right) \mathcal{I} m(\tilde{\eta}(z))-\mathcal{I} m\left(\eta^{4}(z)\right) \operatorname{Re}(\tilde{\eta}(z))\right]-y \mathcal{R} e\left(\eta^{4}(z)\right), \\
\left\langle\omega_{0}, \omega_{2}\right\rangle & =4 y^{2}\left[\mathcal{R} e\left(\eta^{4}(z)\right) \operatorname{Re}(\tilde{\eta}(z))+\mathcal{I} m\left(\eta^{4}(z)\right) \mathcal{I} m(\tilde{\eta}(z))\right]-y \mathcal{I} m\left(\eta^{4}(z)\right) .
\end{aligned}
$$

\section{Left Brownian motion on $G$}

The Brownian motion $g_{s}=g\left(z_{s}, \theta_{s}\right)=g\left(y_{s}, x_{s}, \theta_{s}\right)$ on $G$ has infinitesimal generator $\frac{1}{2} \Delta^{a}$ and is the left Brownian motion solving the Stratonovitch stochastic differential equation

$$
d g_{s}=g_{s} \circ\left(\lambda d Y_{s}+\alpha d X_{s}+\kappa a d W_{s}\right),
$$

where $\left(Y_{s}, X_{s}, W_{s}\right)$ denotes a 3 -dimensional standard Brownian motion.

Since a direct calculation shows that

$$
g(z, \theta)^{-1} d g(z, \theta)=(\sin \theta d y+\cos \theta d x) \frac{\lambda}{y}+(\cos \theta d y-\sin \theta d x) \frac{\alpha}{y}+(y d \theta+d x) \frac{\kappa}{y},
$$

we get the differential system

$$
\begin{aligned}
& d y_{s}=y_{s} \sin \theta_{s} \circ d Y_{s}+y_{s} \cos \theta_{s} \circ d X_{s}=y_{s} \sin \theta_{s} d Y_{s}+y_{s} \cos \theta_{s} d X_{s}, \\
& d x_{s}=y_{s} \cos \theta_{s} \circ d Y_{s}-y_{s} \sin \theta_{s} \circ d X_{s}=y_{s} \cos \theta_{s} d Y_{s}-y_{s} \sin \theta_{s} d X_{s}, \\
& d \theta_{s}=a d W_{s}-\cos \theta_{s} \circ d Y_{s}+\sin \theta_{s} \circ d X_{s}=a d W_{s}-\cos \theta_{s} d Y_{s}+\sin \theta_{s} d X_{s} .
\end{aligned}
$$

Setting $d U_{s}:=\sin \theta_{s} d Y_{s}+\cos \theta_{s} d X_{s}$ and $d V_{s}:=\cos \theta_{s} d Y_{s}-\sin \theta_{s} d X_{s}$, we get a standard 3-dimensional Brownian motion $\left(U_{s}, V_{s}, W_{s}\right)$ such that

$$
d y_{s}=y_{s} d U_{s}, d x_{s}=y_{s} d V_{s}, d \theta_{s}=a d W_{s}-d V_{s} .
$$


Hence we see that the projection of our Brownian motion $g_{s}=\left(y_{s}, x_{s}, \theta_{s}\right)$ on the hyperbolic plane $\mathbb{H}^{2}$, that is to say on the Iwasawa coordinates $(y, x)$, is simply the standard hyperbolic Brownian motion of $\mathbb{H}^{2}$, and that the angular component $\left(\theta_{s}\right)$ is just a real Brownian motion with variance $\left(1+a^{2}\right)$.

Remark 2 The degenerate limit-case $a=0$ is quite possible for the left Brownian motion $\left(g_{s}\right)$. It corresponds to the Carnot degenerate metric on $G$, and to the horizontal left Brownian motion on $G$, associated with the Levi-Civita connexion on $\mathbb{H}^{2}$.

\section{Three martingales}

Let us denote by

$$
M_{t}^{j}:=\int_{g[0, t]} \omega_{j}, \quad j \in\{0,1,2\},
$$

the 3 martingales obtained by integrating the 3 harmonic forms $\omega_{j}$ along the paths of the left Brownian motion $\left(g_{s}\right)$. Let us introduce also

$$
M_{t}:=M_{t}^{1}+\sqrt{-1} M_{t}^{2}=\int_{g[0, t]} \eta^{4}(z) d z
$$

which by Corollary 1 is a conformal martingale. Note that we may as well consider the Brownian motion $\left(g_{s}\right)$ as living on $G$ or on $G / \Gamma^{\prime}$.

Section 7 and Theorem 1 show that

$$
\begin{aligned}
& M_{t}^{0}=a W_{t}+\int_{0}^{t}\left(4 \mathcal{R} e \tilde{\eta}\left(z_{s}\right) y_{s} d U_{s}+\left(4 \mathcal{I} m \tilde{\eta}\left(z_{s}\right) y_{s}-1\right) d V_{s}\right) \\
& M_{t}^{1}=\int_{0}^{t}\left(\mathcal{R} e \eta^{4}\left(z_{s}\right) y_{s} d V_{s}-\mathcal{I} m \eta^{4}\left(z_{s}\right) y_{s} d U_{s}\right) \\
& M_{t}^{2}=\int_{0}^{t}\left(\mathcal{I} m \eta^{4}\left(z_{s}\right) y_{s} d V_{s}+\mathcal{R} e \eta^{4}\left(z_{s}\right) y_{s} d U_{s}\right)
\end{aligned}
$$

and then

$$
M_{t}=\int_{0}^{t} \eta^{4}\left(z_{s}\right) y_{s} d\left(V_{s}+\sqrt{-1} U_{s}\right) .
$$

Lemma 5 The law of $\left(M_{t}^{1}, M_{t}^{2}\right) / \sqrt{t}$ converges towards the centered Gaussian law with covariance matrix equal to the unit matrix multiplied by the variance $\frac{3}{\pi} \int_{D_{0}}|\eta(z)|^{8} d x d y$. 
Proof. By the above, we have some complex Brownian motion $\left(\tilde{Z}_{s}\right)$ such that

$$
M_{t}=\tilde{Z}\left(\langle M\rangle_{t}\right)=\tilde{Z}\left(\int_{0}^{t} y_{s}^{2}\left|\eta\left(z_{s}\right)\right|^{8} d s\right)
$$

and then by scaling, we have the following identity in law (for each $t>0$ ):

$$
t^{-\frac{1}{2}} M_{t} \equiv \tilde{Z}\left(\langle M\rangle_{t} / t\right)=\tilde{Z}\left(t^{-1} \int_{0}^{t} y_{s}^{2}\left|\eta\left(z_{s}\right)\right|^{8} d s\right)
$$

which by ergodicity converges to

$$
\tilde{Z}\left(\int_{G / \Gamma^{\prime}} y^{2}|\eta(z)|^{8} d \mu\right)=\tilde{Z}\left(\int_{D}|\eta(z)|^{8} \frac{d x d y}{2 \pi}\right)=\tilde{Z}\left(\frac{3}{\pi} \int_{D_{0}}|\eta(z)|^{8} d x d y\right) .
$$

Then let us consider, for any $r>0$, the martingale

$$
N_{t}^{r}:=\frac{\pi}{3} \int_{0}^{t} 1_{\left\{\tilde{y}_{s}>r\right\}} y_{s} d V_{s}=\frac{\pi}{3} \int_{0}^{t} 1_{\left\{\tilde{y}_{s}>r\right\}} d x_{s}
$$

where $\tilde{y}$ is the height in the cusp (defined in section 5).

Now using that $\omega_{0}$ is bounded in the compact $\left\{\tilde{y}_{s} \leq r\right\}$ and using Lemma $3, i i i)$, observe that $M_{t}^{0}-N_{t}^{r}$ is a martingale with bounded quadratic variation, so that as $t \rightarrow \infty,\left(M_{t}^{0}-N_{t}^{r}\right) / \sqrt{t}$ converges in law and $\left(M_{t}^{0}-N_{t}^{r}\right) / t$ goes to 0 in $L^{2}$-norm.

Hence $M_{t}^{0} / t$ behaves as $N_{t}^{r} / t$, which depends only on the hyperbolic Brownian motion $\left(z_{s}\right)$, as $M_{t}$.

\section{Asymptotic Brownian windings in $G / \Gamma^{\prime}$}

The following theorem describes the asymptotic Brownian windings in $G / \Gamma^{\prime}$.

Theorem 2 As $t \rightarrow \infty$,

$$
\left(\frac{M_{t}^{0}}{t}, \frac{M_{t}^{1}}{\sqrt{t}}, \frac{M_{t}^{2}}{\sqrt{t}}\right) \quad \text { converges in law towards }\left(\mathcal{C}, \mathcal{N}^{1}, \mathcal{N}^{2}\right),
$$

where the variables $\mathcal{C}, \mathcal{N}^{1}, \mathcal{N}^{2}$ are independent, $\mathcal{C}$ is Cauchy with parameter $\frac{1}{2}$, and $\mathcal{N}^{1}, \mathcal{N}^{2}$ are centred Gaussian with variance $\frac{3}{\pi} \int_{D_{0}}|\eta(z)|^{8} d x d y$.

Observe the irrelevance of the parameter $a$ in this theorem, which is valid as well in the degenerate case $a=0$. The reason is that $a$ was initially the inverse norm of $\mathcal{L}_{\kappa}=\frac{\partial}{\partial \theta}$, which does not concern $\omega_{1}$ and $\omega_{2}$, and which in $\omega_{0}$ contributes only to a second order term. Technically, in the proof of Theorem 2 below $M_{t}^{0} / t$ is firstly replaced by $N_{t}^{r} / t$ (defined at the end of Section 8) which, as $M_{t}$, does not depend on the real parameter $a$. 
Remark 3 Theorem 2 is true as well for all finite dimensional marginals: as $t \rightarrow \infty, \quad\left(M_{c_{j} t}^{0} / t, M_{c_{j} t}^{1} / \sqrt{t}, M_{c_{j} t}^{2} / \sqrt{t}\right), 1 \leq j \leq N$, for any given $N \in \mathbb{N}^{*}$ and $0<c_{1}<\cdots<c_{N}$, converge jointly towards $\left(\mathcal{C}_{c_{j}}, \mathcal{N}_{c_{j}}^{1}, \mathcal{N}_{c_{j}}^{2}\right)$, where the processes $\mathcal{C}, \mathcal{N}^{1}, \mathcal{N}^{2}$ are independent, started from $0, \mathcal{C}$ is Cauchy with parameter $\frac{1}{2}$, and $\mathcal{N}^{1}, \mathcal{N}^{2}$ are real Brownian with variance $\frac{3}{\pi} \int_{D_{0}}|\eta(z)|^{8} d z$.

Proof of this remark 3 is somewhat more tedious than the proof of Theorem 2 , but without notable additional difficulty (but notational). So it will be omitted.

\section{Proof of Theorem 2}

Let us split this involved proof in several items.

Note that a large part of the arguments and techniques employed below already appeared more or less in the union of [2], [4], [5], and [6], but none of these articles contain them almost all, and the major difficulty here is to establish the asymptotic independence of regular and singular windings, that is to say the independence of $\mathcal{C}$ and $\left(\mathcal{N}^{1}, \mathcal{N}^{2}\right)$ in the theorem. Moreover such a question of independence seems not to have been yet dealed with, except in [7] by means of a coding method concerning the geodesic flow on a surface. From a probabilistic and technical point of view, this could be the main interest of the present proof.

\subsection{Excursions near the cusp}

Fix $r, q>0$, and set $\tau:=\min \left\{s>0 \mid \tilde{y}_{s}>r+q\right\}, \sigma:=\min \left\{s>0 \mid \tilde{y}_{s}<r\right\}$, $\tau_{0}:=0$, and for $n \in \mathbb{N}: \sigma_{n}:=\tau_{n}+\sigma \circ \Theta_{\tau_{n}}, \tau_{n+1}:=\sigma_{n}+\tau \circ \Theta_{\sigma_{n}}$.

Note that $\left[\tau_{n}, \sigma_{n}\right]$ is the $n$-th discretized excursion interval near the cusp (cut at level $r$ ).

Let us also set $\zeta_{n}:=\max \left\{s \leq \sigma_{n} \mid \tilde{y}_{s} \geq r+q\right\}$.

Denote by $C_{t}^{r}:=\max \left\{n \in \mathbb{N} \mid \zeta_{n} \leq t\right\}$ the number of complete discrete excursions near the cusp performed till time $t$. This is an additive functional, and then $C_{t}^{r} / t$ converges almost surely as $t \rightarrow \infty$, by the ergodic theorem, towards some constant $\varrho$.

Note that we shall finally let $r$ and $q$ go to $\infty$, in such a way that $q / r \rightarrow 0$ (taking for example $q=\sqrt{r}$ ).

Observe then that we have $\lim _{N \rightarrow \infty} \sum_{n=1}^{N}\left(\sigma_{n}-\tau_{n}\right) / N=\mathbb{E}\left(\sigma_{1}-\tau_{1}\right)$ almost surely, since the irrelevance of the entrance points of our excursions (coming from the fact that $\left(y_{s}\right)$ is an autonomous diffusion) and the Markov property imply the independence of the different variables $\left(\sigma_{n}-\tau_{n}\right)$. 
Observe also that

$$
\int_{0}^{t} 1_{\left\{\tilde{y}_{s}>r+q\right\}} d s \leq \sum_{n=0}^{1+C_{t}^{r}}\left(\sigma_{n}-\tau_{n}\right) \quad \text { and } \quad \int_{0}^{t} 1_{\left\{\tilde{y}_{s}>r\right\}} d s \geq \sum_{n=1}^{C_{t}^{r}}\left(\sigma_{n}-\tau_{n}\right) .
$$

Thus the ergodic theorem yields:

$$
\frac{3}{\pi(r+q)}=\mu[\{\tilde{y}>r+q\}] \leq \varrho \times \mathbb{E}\left(\sigma_{1}-\tau_{1}\right) \leq \mu[\{\tilde{y}>r\}]=\frac{3}{\pi r} .
$$

\subsection{Laws of an excursion}

We need the duration and winding law of the typical excursion. We proceed more or less as in [4].

Fix $c \in \mathbb{R}_{+}$and observe by direct application of Itô's formula that $e^{-c s} \times\left(y_{s}\right)^{(1-\sqrt{1+8 c}) / 2}$ is a bounded martingale. Hence the optional sampling theorem gives

$$
\mathbb{E}_{r+q}\left[e^{-c \sigma}\right]=\left(1+\frac{q}{r}\right)^{(1-\sqrt{1+8 c}) / 2}
$$

This implies that $\mathbb{E}\left[\sigma_{n}-\tau_{n}\right]=2 \log \left(1+\frac{q}{r}\right) \quad$ for any $n \in \mathbb{N}^{*}$.

Note that this and Section 10.1 imply that

$$
h(r, q):=\frac{3}{2 \pi(r+q) \log \left(1+\frac{q}{r}\right)} \leq \varrho \leq h^{\prime}(r, q):=\frac{3}{2 \pi r \log \left(1+\frac{q}{r}\right)},
$$

and therefore that $\lim _{q / r \rightarrow 0} \varrho q=3 /(2 \pi)$.

Fix then $b \in \mathbb{R}$, and observe by direct application of Itô's formula that $\exp \left(-|b| y_{s}-\frac{b^{2}}{2} \int_{0}^{s} y_{t}^{2} d t\right)$ is a bounded martingale, and then we have by the optional sampling theorem

$$
\mathbb{E}_{r+q}\left[\exp \left(-\frac{b^{2}}{2} \int_{0}^{\sigma} y_{t}^{2} d t\right)\right]=e^{-|b| q}
$$

Note that this shows that the variables $\left\{\int_{\tau_{n}}^{\sigma_{n}} y_{s}^{2} d s \mid n \geq 1\right\}$ are independent and stable with parameter $\frac{1}{2}$. Moreover using the existence of a standard real Brownian motion $\left(w_{s}\right)$ independent of $\left(y_{s}\right)$ such that during each excursion in $\{\tilde{y}>r\}$ we have $x_{s}=x_{0}+w\left(\int_{0}^{s} \tilde{y}_{t}^{2} d t\right)$, we deduce that the variables $\left\{\varphi_{n}:=\int_{\tau_{n}}^{\sigma_{n}} d x_{s} \mid n \geq 1\right\}$ are independent and Cauchy with parameter $q$. 


\subsection{Approximation of regular windings}

For $j=1,2$, consider

$$
M_{t}^{j}(r):=\int_{0}^{t} 1_{\left\{\tilde{y}_{s} \leq r\right\}} d M_{s}^{j} .
$$

It is clear (see Lemma 5) that $\left(M_{t}^{j}-M_{t}^{j}(r)\right) / \sqrt{t}$ converges in law, with quadratic variation going almost surely to $\mathcal{O}(\mu(\{\tilde{y}>r\}))=\mathcal{O}(1 / r)$; so that $\left(M_{t}^{j}-M_{t}^{j}(r)\right) / t$ converges uniformly to 0 in probability as $r \rightarrow \infty$.

For $\ell \in \mathbb{R}^{2}$ and $n \in \mathbb{N}^{*}$, set

$$
J_{n}^{\ell}:=\int_{\sigma_{n}}^{\tau_{n+1}}\left(\ell_{1} d M_{s}^{1}(r)+\ell_{2} d M_{s}^{2}(r)\right)
$$

so that we have

$$
\begin{aligned}
\ell_{1} M_{t}^{1}(r)+\ell_{2} M_{t}^{2}(r)= & \ell_{1} M_{\tau_{1}}^{1}(r)+\ell_{2} M_{\tau_{1}}^{2}(r) \\
& +\sum_{n=1}^{C_{t}^{r}} J_{n}^{\ell}-\int_{t}^{\tau_{C_{t}+1}} 1_{\left\{\tilde{y}_{s} \leq r\right\}}\left(\ell_{1} d M_{s}^{1}+\ell_{2} d M_{s}^{2}\right) .
\end{aligned}
$$

Now $\left(\ell_{1} M_{\tau_{1}}^{1}(r)+\ell_{2} M_{\tau_{1}}^{2}(r)\right) / \sqrt{t}$ goes to 0 almost surely as $t \rightarrow \infty$, and $R_{t}:=\left\langle\frac{1}{\sqrt{t}} \int_{t}^{\tau_{C_{t}^{r}+1}} 1_{\left\{\tilde{y}_{s} \leq r\right\}}\left(\ell_{1} d M_{s}^{1}+\ell_{2} d M_{s}^{2}\right)\right\rangle=\frac{1}{t} \mathcal{O}\left(\sup \left\{\left(\tau_{n+1}-\sigma_{n}\right) \mid n \leq C_{t}^{r}\right\}\right)$, so that for any $\varepsilon^{\prime}>0$

$$
\begin{aligned}
\mathbb{P}\left(R_{t}>\varepsilon^{\prime}\right) & \leq \mathbb{P}\left(C_{t}^{r}>2 \varrho t\right)+\mathbb{P}\left(\sup \left\{\left(\tau_{n+1}-\tau_{n}\right) \mid n \leq 2 \varrho t\right\} \geq \varepsilon^{\prime} t\right) \\
& \leq o(1)+2 \varrho t \mathbb{P}\left(\tau_{2}-\sigma_{1} \geq \varepsilon^{\prime} t\right)=o(1)+\mathcal{O}(1 / t),
\end{aligned}
$$

since $\left(\tau_{2}-\sigma_{1}\right)$ is square integrable, as exit time of the compact $\{\tilde{y} \leq r+q\}$. This shows that

$$
\int_{t}^{\tau_{C_{t}^{r}+1}} 1_{\left\{\tilde{y}_{s} \leq r\right\}}\left(\ell_{1} d M_{s}^{1}+\ell_{2} d M_{s}^{2}\right) / \sqrt{t}
$$

goes to 0 in probability as $t \rightarrow \infty$.

Then similarly

$$
\begin{aligned}
\mathbb{P} & \left.\left\langle\left(\sum_{n=1}^{C_{t}^{r}} J_{n}^{\ell}-\sum_{n=1}^{[\varrho t]} J_{n}^{\ell}\right) / \sqrt{t}\right\rangle>\varepsilon^{\prime}\right] \leq \\
& \leq \mathbb{P}\left[\left|C_{t}^{r} / t-\varrho\right|>\varepsilon^{\prime}\right]+\mathbb{P}\left[\mathcal{O}\left(\sum_{n=\left[\left(\varrho-\varepsilon^{\prime}\right) t\right]}^{\left[\left(\varrho+\varepsilon^{\prime}\right) t\right]}\left(\tau_{n+1}-\sigma_{n}\right)\right)>\varepsilon^{\prime} t\right] \\
& =o(1)+\mathcal{O}(1 / t)
\end{aligned}
$$


provided we can establish that the correlations between the variables $\left(\tau_{n+1}-\sigma_{n}\right)$ decay exponentially. To this end, observe that by Markov property and Schwarz inequality

$$
\begin{aligned}
\left|\operatorname{Cov}\left\{\left(\tau_{n+m+1}-\sigma_{n+m}\right),\left(\tau_{n+1}-\sigma_{n}\right)\right\}\right|^{2} \leq & \mathbb{E}\left[\left(\tau_{n+1}-\sigma_{n}\right)^{2}\right] \\
& \times \mathbb{E}\left[\left|\mathbb{E}_{z_{\sigma_{n+m}}}(\tau)-\mathbb{E}_{z_{\sigma_{n}}}(\tau)\right|^{2}\right]
\end{aligned}
$$

showing that we shall be done if we prove that $\mathbb{E}_{z_{\sigma_{n}}}(\tau)$ converges exponentially fast in $L^{2}$-norm as $n \rightarrow \infty$. Now viewing the ergodic stationary historical process $\left(z_{s} \mid-\infty<s \leq t\right)$ as a suspended flow under the function $\tau$, we may apply Ambrose's Theorem to deduce that the induced Markov chain $\left(z_{\sigma_{n}}, z_{\tau_{n+1}}\right)$ is stationary and ergodic under the so-called Palm invariant probability measure $\chi$ induced by $\mu$. See [16, Exposés I and II]. $\chi$ being clearly compactly supported, the transition operator of this Markov chain has a spectral gap in $L^{2}(\chi)$, from which our exponential decay directly follows.

Hence we have shown that

$$
\frac{1}{\sqrt{t}}\left(\sum_{n=1}^{C_{t}^{r}} J_{n}^{\ell}-\sum_{n=1}^{[\varrho t]} J_{n}^{\ell}\right)
$$

goes to 0 in probability as $t \rightarrow \infty$.

Therefore we have proved that, as $t \rightarrow \infty,\left(\ell_{1} M_{t}^{1}(r)+\ell_{2} M_{t}^{2}(r)\right) / \sqrt{t}$ behaves in probability as $\sum_{n=1}^{[\varrho t]} J_{n}^{\ell} / \sqrt{t}$.

\subsection{Approximation of singular windings}

Recall from Section 8 that $\left(M_{t}^{0}-N_{t}^{r}\right) / t$ goes to 0 in $L^{2}$-norm, and write

$$
N_{t}^{r}=\frac{\pi}{3} \int_{0}^{\sigma_{0}} 1_{\left\{\tilde{y}_{s}>r\right\}} d x_{s}+\frac{\pi}{3} \sum_{n=1}^{C_{t}^{r}} \int_{\tau_{n}}^{\sigma_{n}} d x_{s}+\frac{\pi}{3} \int_{\tau_{t \wedge C_{t}^{r}+1}^{t}}^{t} d x_{s}+\int_{0}^{t} \mathcal{O}(1) d V_{s} .
$$

It is obvious that

$$
\int_{0}^{\sigma_{0}} 1_{\left\{\tilde{y}_{s}>r\right\}} d x_{s} / t
$$

goes to 0 almost surely, and that

$$
\int_{0}^{t} \mathcal{O}(1) d V_{s} / t
$$

goes to 0 in $L^{2}$-norm, as $t \rightarrow \infty$. 
Then

$$
\mathbb{P}\left[\int_{\tau_{t \wedge C_{t}^{r}+1}^{t}}^{t} d x_{s} \neq 0\right]=\mathbb{P}\left[\tilde{y}_{t}>r+q\right]=\mathcal{O}(\mu[\{\tilde{y}>r+q\}])=\mathcal{O}(1 / r) .
$$

Moreover, using that the $\varphi_{n}=\int_{\tau_{n}}^{\sigma_{n}} d x_{s}, n \in \mathbb{N}^{*}$, are independent and Cauchy with parameter $q$, as shown in Section 10.2 above, and denoting by $\mathcal{C}$ a right continuous Cauchy process with parameter $q$, we have for any $\varepsilon^{\prime}, \varepsilon^{\prime \prime}>0$ :

$$
\begin{aligned}
& \mathbb{P}\left[\left|\sum_{n=1}^{C_{t}^{r}} \varphi_{n}-\sum_{n=1}^{[\varrho t]} \varphi_{n}\right| / t>\varepsilon^{\prime}\right]-\mathbb{P}\left[\left|C_{t}^{r} / t-\varrho\right|>\varepsilon^{\prime \prime}\right] \leq \\
& \quad \leq 2 \mathbb{P}\left[\max _{0 \leq k \leq \varepsilon^{\prime \prime} t}\left|\sum_{n=[\varrho t]}^{[\varrho t]+k} \varphi_{n}\right|>\varepsilon^{\prime} t\right] \leq \mathbb{P}\left[\sup _{0 \leq s \leq \varepsilon^{\prime \prime} t}\left|\mathcal{C}_{s}\right|>\varepsilon^{\prime} t\right]=\mathbb{P}\left[\sup _{0 \leq s \leq \varepsilon^{\prime \prime}}\left|\mathcal{C}_{s}\right|>\varepsilon^{\prime}\right],
\end{aligned}
$$

showing that

$$
\limsup _{t \rightarrow \infty} \mathbb{P}\left[\left|\sum_{n=1}^{C_{t}^{r}} \varphi_{n}-\sum_{n=1}^{[\varrho t]} \varphi_{n}\right| / t>\varepsilon^{\prime}\right] \leq \mathbb{P}\left[\sup _{0 \leq s \leq \varepsilon^{\prime \prime}}\left|\mathcal{C}_{s}\right|>\varepsilon^{\prime}\right]
$$

whence the convergence to 0 in probability of $\left|\sum_{n=1}^{C_{t}^{r}} \varphi_{n}-\sum_{n=1}^{[\varrho t]} \varphi_{n}\right| / t \quad$ by letting $\varepsilon^{\prime \prime}$ decrease to 0 . This method was already in [5].

So we have proved that, as $t \rightarrow \infty, M_{t}^{0} / t$ behaves in probability as $\frac{\pi}{3} \sum_{n=1}^{[\varrho t]} \varphi_{n} / t$, and this with probability $1-\mathcal{O}(1 / r)$.

\subsection{Conditional independence}

So far, we have established that for any $\left(\ell, \ell^{\prime}\right) \in \mathbb{R}^{2} \times \mathbb{R}$

$$
\begin{aligned}
A_{\ell}: & =\lim _{t \rightarrow \infty} \mathbb{E}\left[\exp \left(\sqrt{-1}\left[\left(\ell_{1} M_{t}^{1}+\ell_{2} M_{t}^{2}\right) / \sqrt{t}+\ell^{\prime} M_{t}^{0} / t\right]\right)\right] \\
& =\lim _{r \rightarrow \infty} \lim _{t \rightarrow \infty} \mathbb{E}\left[\exp \left(\sqrt{-1}\left[\left(\ell_{1} M_{t}^{1}(r)+\ell_{2} M_{t}^{2}(r)\right) / \sqrt{t}+\ell^{\prime} N_{t}^{r} / t\right]\right)\right] \\
& =\lim _{r \rightarrow \infty}\left(\lim _{t \rightarrow \infty} \mathbb{E}\left[\exp \left(\sqrt{-1}\left[\sum_{n=1}^{[\varrho t]} J_{n}^{\ell} / \sqrt{t}+\frac{\ell^{\prime} \pi}{3} \sum_{n=1}^{[\varrho t]} \varphi_{n} / t\right]\right)\right]+\mathcal{O}(1 / r)\right) \\
& =\lim _{r \rightarrow \infty} \lim _{t \rightarrow \infty} \mathbb{E}\left\{\mathbb{E}^{\mathcal{F}}\left[\exp \left(\sqrt{-1}\left[\sum_{n=1}^{[\varrho t]} J_{n}^{\ell} / \sqrt{t}+\frac{\ell^{\prime} \pi}{3 t} \sum_{n=1}^{[\varrho t]} \varphi_{n}\right]\right)\right]\right\},
\end{aligned}
$$

denoting by $\mathcal{F}$ the $\sigma$-algebra generated by the variables $\left\{\left(z_{\sigma_{n}}, z_{\tau_{n+1}}\right) \mid n \in \mathbb{N}\right\}$. 
Now the strong Markov property insures the conditional independence of the variables $\left\{J_{n}^{\ell}, \varphi_{n} \mid n \in \mathbb{N}\right\}$. Therefore

$$
\begin{aligned}
& A_{\ell}=\lim _{r \rightarrow \infty} \lim _{t \rightarrow \infty} \mathbb{E}\left\{\mathbb{E}^{\mathcal{F}}\left[\exp \left(\sqrt{-1} \sum_{n=1}^{[\varrho t]} J_{n}^{\ell / \sqrt{t}}\right)\right] \mathbb{E}^{\mathcal{F}}\left[\exp \left(\sqrt{-1}\left(\ell^{\prime} \pi / 3 t\right) \sum_{n=1}^{[\varrho t]} \varphi_{n}\right)\right]\right\} \\
& =\lim _{r \rightarrow \infty} \lim _{t \rightarrow \infty} \mathbb{E}\left\{\prod_{n=1}^{[\varrho t]} \mathbb{E}^{\mathcal{F}}\left[e^{\sqrt{-1} J_{n}^{\ell / \sqrt{t}}}\right] \times \prod_{n=1}^{[\varrho t]} \mathbb{E}^{\mathcal{F}}\left[e^{\sqrt{-1}\left(\ell^{\prime} \pi / 3 t\right) \varphi_{n}}\right]\right\} \\
& =\lim _{r \rightarrow \infty} \lim _{t \rightarrow \infty} \mathbb{E}\left\{\prod_{n=1}^{[\varrho t]} \mathbb{E}_{z_{\sigma_{n}}}^{z_{\tau_{n+1}}}\left[e^{\sqrt{-1} J_{n}^{\ell / \sqrt{t}}}\right] \times \prod_{n=1}^{[\varrho t]} \mathbb{E}_{z_{\tau_{n}}}^{z_{\sigma_{n}}}\left[e^{\sqrt{-1}\left(\ell^{\prime} \pi / 3 t\right) \varphi_{n}}\right]\right\} .
\end{aligned}
$$

\subsection{Getting rid of the conditioning}

We need to get rid of the above conditioning by $\mathcal{F}$. We first follow the argument of ([2], Lemma 12). Setting $Y:=\int_{\tau_{1}}^{\sigma_{1}} y_{s}^{2} d s$ and using again that $x_{s}=x_{0}+w\left(\int_{0}^{s} y_{t}^{2} d t\right)$ during each excursion, we have for any real $b$ :

$$
\mathbb{E}_{z_{\tau_{n}}}^{z_{\sigma_{n}}}\left[e^{\sqrt{-1} b \varphi_{n}}\right]=\mathbb{E}\left[e^{\sqrt{-1} b w(Y)} \mid w(Y) \operatorname{modulo} 1\right]
$$

and for any real $c$ and positive $\varepsilon^{\prime}$ :

$$
\begin{aligned}
\mathbb{E}\left[e^{\sqrt{-1} b w(Y) \mid w(Y) \in] c, c+\varepsilon^{\prime}[+\mathbb{Z}]-1}\right. & \mathbb{E}\left[\left(e^{\sqrt{-1} b w(Y)}-1\right) \times \sum_{k \in \mathbb{Z}} 1_{\left\{c<w(Y)-k<c+\varepsilon^{\prime}\right\}}\right] \\
= & \frac{\mathbb{E}\left[\sum_{k \in \mathbb{Z}} 1_{\left\{c<w(Y)-k<c+\varepsilon^{\prime}\right\}}\right]}{\mathbb{E}\left[(2 \pi Y)^{-1 / 2} \int_{\mathbb{R}} \sum_{k \in \mathbb{Z}}\left(e^{\sqrt{-1} b x}-1\right) e^{-x^{2} /(2 Y)} 1_{\left\{c<x-k<c+\varepsilon^{\prime}\right\}} d x\right]} \\
= & \frac{\mathbb{E}\left[(2 \pi Y)^{-1 / 2} \int_{\mathbb{R}} \sum_{k \in \mathbb{Z}} e^{-x^{2} /(2 Y)} 1_{\left\{c<x-k<c+\varepsilon^{\prime}\right\}} d x\right]}{\mathbb{E}\left[(2 \pi Y)^{-1 / 2} \int_{c}^{c+\varepsilon^{\prime}} \sum_{k \in \mathbb{Z}}\left(e^{\sqrt{-1} r(x+k)}-1\right) e^{-(x+k)^{2} /(2 Y)} d x\right]} \\
= & \frac{\left.\mathbb{E}^{-1 / 2} \int_{c}^{c+\varepsilon^{\prime}} \sum_{k \in \mathbb{Z}} e^{-(x+k)^{2} /(2 Y)} d x\right]}{}
\end{aligned}
$$


Then

$$
\begin{aligned}
& \sup \left\{\left|e^{\sqrt{-1} b k}-1\right| \times e^{-k^{2} /(2 Y)} \mid k \in \mathbb{R}\right\} \\
& \leq \sup \left\{\min \{2,|b k|\} \times e^{-k^{2} /(2 Y)} \mid k \in \mathbb{R}\right\} \\
& =\max \left\{\max \left\{e^{-k^{2} /(2 Y)}|k \geq 2 /| b \mid\right\} ; \max \left\{|b k| \times e^{-k^{2} /(2 Y)}|0 \leq k \leq 2 /| b \mid\right\}\right\} \\
& =\max \left\{e^{-2\left(b^{2} Y\right)^{-1}} ; \min \{2,|b| \sqrt{Y}\} \times \exp \left[-\min \left\{1,4 b^{-2} / Y\right\} / 2\right]\right\} \\
& =2 e^{-2\left(b^{2} Y\right)^{-1}} 1_{\left\{Y>4 b^{-2}\right\}}+|b| \sqrt{Y} \times e^{-1 / 2} 1_{\left\{Y \leq 4 b^{-2}\right\}} \\
& \leq|b| \sqrt{Y} .
\end{aligned}
$$

Hence we can replace the Riemannian sum above by a Riemannian integral + an error term:

$$
\begin{aligned}
\sum_{k \in \mathbb{Z}}\left(e^{\sqrt{-1} b(x+k)}\right. & -1) e^{-(x+k)^{2} /(2 Y)} \\
& =\int_{\mathbb{R}}\left(e^{\sqrt{-1} b(x+k)}-1\right) e^{-(x+k)^{2} /(2 Y)} d k+\mathcal{O}(|b| \sqrt{Y}) \\
& =\left(e^{-b^{2} Y / 2}-1\right) \sqrt{2 \pi Y}+\mathcal{O}(|b| \sqrt{Y})
\end{aligned}
$$

Therefore we obtain for all $c$ and $\varepsilon^{\prime}>0$ (with a uniform $\mathcal{O}$ ):

$$
\begin{gathered}
\mathbb{E}\left(e^{\sqrt{-1} b W(Y) \mid} w(Y) \in\right] c, c+\varepsilon^{\prime}[+\mathbb{Z})-1=\frac{\varepsilon^{\prime} \times \mathbb{E}\left[\left(e^{-b^{2} Y / 2}-1\right)+\mathcal{O}(|b|)\right]}{\varepsilon^{\prime} \times\left(1+\mathcal{O}\left(\mathbb{E}\left(Y^{-1 / 2}\right)\right)\right)} \\
=\left[e^{-|b| q}-1+\mathcal{O}(|b|)\right] /(1+\mathcal{O}(1 / q))
\end{gathered}
$$

since $\mathbb{E}\left[e^{-b^{2} Y / 2}\right]=e^{-|b| q}$, which in turn implies

$$
\mathbb{E}\left(Y^{-1 / 2}\right)=\frac{1}{\sqrt{2 \pi}} \int_{\mathbb{R}} e^{-|b| q} d b=\frac{1}{q} .
$$

This proves that

$$
\begin{aligned}
\mathbb{E}_{z_{\tau_{n}}}^{z_{\sigma_{n}}}\left[e^{\sqrt{-1} b \varphi_{n}}\right] & =1-\left(1+\mathcal{O}_{n}(1 / q)\right) \times\left(1-e^{-|b| q}+\mathcal{O}_{n}(|b|)\right) \\
& =1-\left(1+\mathcal{O}_{n}(1) / q\right)|b| q,
\end{aligned}
$$

with $\mathcal{O}_{n}(1)$ denoting a uniformly bounded function of $z_{\sigma_{n}}$. 


\subsection{End of the proof of Theorem 2}

Let us apply the result of the preceding section 10.6 for large $q$ and $r / q$, and for $b=\ell^{\prime} \pi / 3 t$, with $t \rightarrow \infty$. We get from the above and from Birkhoff's ergodic Theorem applied to the Markov chain $\left(z_{\sigma_{n}}\right)$ (via the sequence $\left.\mathcal{O}_{n}(1)\right)$ :

$$
\begin{aligned}
\prod_{n=1}^{[\varrho t]} \mathbb{E}_{z_{\tau_{n}}}^{z_{\sigma_{n}}}\left[e^{\left.\sqrt{-1}\left(\ell^{\prime} \pi / 3 t\right) \varphi_{n}\right]}\right. & =\prod_{n=1}^{[\varrho t]}\left[1-\left(1+\mathcal{O}_{n}(1) / q\right)\left|\ell^{\prime}\right| q \pi / 3 t\right] \\
& =\exp \left(-\frac{\left|\ell^{\prime}\right| q \pi}{3 t} \sum_{n=1}^{[\varrho t]}\left(1+\mathcal{O}_{n}(1) / q\right)+o(1)\right) \\
& \stackrel{t \rightarrow \infty}{\longrightarrow} \exp \left(-\frac{\left|\ell^{\prime}\right| \varrho q \pi}{3}(1+\mathcal{O}(1 / q))\right) .
\end{aligned}
$$

Coming back to Section 10.5 and taking for example $q:=\sqrt{r}$, this yields:

$$
\begin{aligned}
A_{\ell}=\lim _{r \rightarrow \infty} \lim _{t \rightarrow \infty} \mathbb{E}\left\{\prod _ { n = 1 } ^ { [ \varrho t ] } \mathbb { E } _ { z _ { \sigma _ { n } } } ^ { z _ { \tau _ { n + 1 } } } \left[e^{\left.\left.\sqrt{-1} J_{n}^{\ell / \sqrt{t}}\right]\right\} \times}\right.\right. \\
\quad \times \exp \left(-\frac{\left|\ell^{\prime}\right| \varrho q \pi}{3}(1+\mathcal{O}(1 / q))\right) \\
=\lim _{r \rightarrow \infty} \lim _{t \rightarrow \infty} \mathbb{E}\left[\exp \left(\frac{\sqrt{-1}}{\sqrt{t}}\left(\ell_{1} M_{t}^{1}(r)+\ell_{2} M_{t}^{2}(r)\right)\right)\right] \times \\
\quad \times \exp \left(-\frac{\left|\ell^{\prime}\right| \varrho q \pi}{3}(1+\mathcal{O}(1 / q))\right) \\
=\lim _{t \rightarrow \infty} \mathbb{E}\left[\exp \left(\frac{\sqrt{-1}}{\sqrt{t}}\left(\ell_{1} M_{t}^{1}+\ell_{2} M_{t}^{2}\right)\right)\right] \times e^{-\left|\ell^{\prime}\right| / 2} \\
=\exp \left(-\frac{3|\ell|^{2}}{\pi} \int_{D_{0}}|\eta(z)|^{8} d z-\frac{\left|\ell^{\prime}\right|}{2}\right)
\end{aligned}
$$

by Section 10.2 and Lemma 5 . This achieves the proof of Theorem 2, since $A_{\ell}$ was defined in Section 10.5 as

$$
\lim _{t \rightarrow \infty} \mathbb{E}\left[\exp \left(\sqrt{-1}\left[\frac{\ell_{1} M_{t}^{1}+\ell_{2} M_{t}^{2}}{\sqrt{t}}+\frac{\ell^{\prime} M_{t}^{0}}{t}\right]\right)\right]
$$




\section{Geodesics of $G$}

\subsection{Description of these geodesics}

The Levi-Civita connexion of $G=P S L_{2}(\mathbb{R})$ equipped with its Riemannian structure $\left(\left(g_{i j}^{a}\right)\right)$ of Section 5 has Christoffel coefficients classically given by

$$
\Gamma_{j k}^{i}=\frac{1}{2}\left(\partial_{j} g_{\ell k}^{a}+\partial_{k} g_{\ell j}^{a}-\partial_{\ell} g_{j k}^{a}\right) \times g_{a}^{\ell i} \quad \text { for } 1 \leq i, j, k \leq 3,
$$

and the geodesics $\left(\gamma_{t}\right)$ are the solutions of the following system:

$$
\frac{d^{2} \gamma_{t}^{i}}{d t^{2}}+\Gamma_{j k}^{i}\left(\gamma_{t}\right) \times \frac{d \gamma_{t}^{j}}{d t} \times \frac{d \gamma_{t}^{k}}{d t}=0 \quad \text { for } 1 \leq i \leq 3
$$

Using the expression of Lemma 4 for $\left(\left(g_{i j}^{a}\right)\right)$, a somewhat tedious but direct computation gives the equation of geodesics $\left(\gamma_{t}=\left(y_{t}, x_{t}, \theta_{t}\right)\right)$ of $G$ in the Iwasawa coordinates by means of the following system:

$$
\begin{aligned}
y_{t}^{\prime \prime}-y_{t}^{\prime 2} / y_{t}+\left(1+a^{-2}\right) x_{t}^{\prime 2} / y_{t}+a^{-2} x_{t}^{\prime} \theta_{t}^{\prime}=0 ; \\
x_{t}^{\prime \prime}-\left(2+a^{-2}\right) y_{t}^{\prime} x_{t}^{\prime} / y_{t}-a^{-2} y_{t}^{\prime} \theta_{t}^{\prime}=0 ; \\
\theta_{t}^{\prime \prime}+\left(1+a^{-2}\right) y_{t}^{\prime} x_{t}^{\prime} / y_{t}^{2}+a^{-2} y_{t}^{\prime} \theta_{t}^{\prime} / y_{t}=0 .
\end{aligned}
$$

Linearly combining the last two equations gives $\theta_{t}^{\prime \prime}=\left(-x_{t}^{\prime} / y_{t}\right)^{\prime}$. Consequently our geodesic system is equivalent to the following, for some real constant $c$.

$$
\begin{aligned}
\theta_{t}^{\prime} & =c-x_{t}^{\prime} / y_{t} ; \\
x_{t}^{\prime \prime}-2 y_{t}^{\prime} x_{t}^{\prime} / y_{t}-c a^{-2} y_{t}^{\prime} & =0 ; \\
y_{t}^{\prime \prime}-y_{t}^{\prime 2} / y_{t}+x_{t}^{\prime 2} / y_{t}+c a^{-2} x_{t}^{\prime} & =0 .
\end{aligned}
$$

Eliminating $\mathrm{ca}^{-2}$ between the last two equations gives some real constant $C$ such that

$$
x_{t}^{\prime 2}+y_{t}^{\prime 2}=C^{2} y_{t}^{2} .
$$

Considering then the $[-1,1]$-valued function $f(t):=y_{t}^{\prime} /\left(C y_{t}\right)$ and eliminating $x_{t}^{\prime}$, we get the equation

$$
f^{\prime}+C\left(1-f^{2}\right) \pm c a^{-2} \sqrt{1-f^{2}}=0 .
$$

Now consider

$$
k:= \pm a^{-2} c / C \quad \text { and } \quad h(z):=\frac{-1}{C} \int_{0}^{z} \frac{d s}{\left(1-s^{2}\right)+k \sqrt{1-s^{2}}} .
$$

Letting apart the simple case when $f$ is constant, and then when $\left(y_{t}, x_{t}\right)$ runs a straight line or is constant, we must have $h \circ f(t)=t-t_{0}$. Changing the variable by $u:=\frac{1-\sqrt{1-s^{2}}}{s} \in[-1,1]$ in the formula for $h$, we get successively the following computations. 
- If $|k|=1: \quad h(z)=\frac{-1 \pm \sqrt{1-z^{2}}}{C z}$ and then

$$
f(t)=\frac{-2 C\left(t-t_{0}\right)}{1+C^{2}\left(t-t_{0}\right)^{2}}=\frac{-1}{C} \frac{d}{d t} \log \left(1+C^{2}\left(t-t_{0}\right)^{2}\right),
$$

whence

$$
y(t)=\frac{C^{\prime}}{1+C^{2}\left(t-t_{0}\right)^{2}}, \quad x(t)=x_{0}+\frac{C C^{\prime}\left(t-t_{0}\right)}{1+C^{2}\left(t-t_{0}\right)^{2}} .
$$

- If $|k|<1: \quad h(z)=\frac{-2}{C \sqrt{1-k^{2}}} \operatorname{argth}\left(\sqrt{\frac{1-k}{1+k}}\left[\frac{1-\sqrt{1-z^{2}}}{z}\right]\right)$ and then

$$
f(t)=\frac{-1}{C} \times \frac{d}{d t} \log \left(\operatorname{ch}\left[C \sqrt{1-k^{2}}\left(t-t_{0}\right)\right]-k\right),
$$

whence

$$
\begin{aligned}
& y(t)=\frac{C^{\prime}}{\operatorname{ch}\left[C \sqrt{1-k^{2}}\left(t-t_{0}\right)\right]-k}, \\
& x(t)=x_{0}+\frac{C^{\prime}}{\sqrt{1-k^{2}}} \times \frac{\operatorname{sh}\left[C \sqrt{1-k^{2}}\left(t-t_{0}\right)\right]}{\operatorname{ch}\left[C \sqrt{1-k^{2}}\left(t-t_{0}\right)\right]-k} .
\end{aligned}
$$

- If $|k|>1: \quad h(z)=\frac{-2}{C \sqrt{k^{2}-1}} \operatorname{arctg}\left(\sqrt{\frac{k-1}{k+1}}\left[\frac{1-\sqrt{1-z^{2}}}{z}\right]\right)$ and then

$$
f(t)=\frac{-1}{C} \times \frac{d}{d t} \log \left(\left|k-\cos \left[C \sqrt{k^{2}-1}\left(t-t_{0}\right)\right]\right|\right),
$$

whence

$$
\begin{aligned}
& y(t)=\frac{C^{\prime}}{k-\cos \left[C \sqrt{k^{2}-1}\left(t-t_{0}\right)\right]}, \\
& x(t)=x_{0}+\frac{C^{\prime}}{\sqrt{k^{2}-1}} \times \frac{\sin \left[C \sqrt{k^{2}-1}\left(t-t_{0}\right)\right]}{k-\cos \left[C \sqrt{k^{2}-1}\left(t-t_{0}\right)\right]} .
\end{aligned}
$$

As a consequence, we see that our geodesics project on the hyperbolic plane $\mathbb{H}^{2}$ in the following way.

- If $|k|=1: \quad\left(x(t)-x_{0}\right)^{2}+\left(y(t)-C^{\prime} / 2\right)^{2}=\left(C^{\prime} / 2\right)^{2}$, and we get an horocycle;

- If $|k|<1: \quad\left(x(t)-x_{0}\right)^{2}+\left(y(t)+\frac{C^{\prime} k}{1-k^{2}}\right)^{2}=\left(\frac{C^{\prime}}{1-k^{2}}\right)^{2}$, and we get an Euclidian circle intersecting $\mathbb{R}$, which is a geodesic if and only if $k=0$;

- If $|k|>1: \quad\left(x(t)-x_{0}\right)^{2}+\left(y(t)-\frac{C^{\prime} k}{k^{2}-1}\right)^{2}=\left(\frac{C^{\prime}}{k^{2}-1}\right)^{2}$, and we get an Euclidian circle totally included in $\mathbb{H}^{2}$ (necessarily $C^{\prime} k>0$ ). This is thus also an hyperbolic circle. 
Finally we recover $\theta(t)$ from the initial equations.

For constant function $f$, we see at once that $\theta^{\prime}(t)$ must be constant.

For $|k|=1$, we find that

$$
\theta(t)=\theta_{0}+\left(1+a^{2}\right) C t-2 \operatorname{arctg}\left(C\left(t-t_{0}\right)\right) .
$$

For $|k|<1$, we find that

$$
\theta(t)=\theta_{0}+C k a^{2} t-2 \operatorname{arctg}\left(\operatorname{th}\left[C \sqrt{1-k^{2}}\left(t-t_{0}\right) / 2\right]\right) .
$$

For $|k|>1$, we find that

$$
\theta(t)=\theta_{0}+C|k| a^{2} t-\sqrt{k^{2}-1} \int_{0}^{C \sqrt{k^{2}-1}\left(t-t_{0}\right)} \frac{d \varphi}{|k|-\cos \varphi} .
$$

In this last case, we observe that we may choose $k>1$, and that using the $2 \pi$ periodicity we have

$$
\int_{0}^{v} \frac{d \varphi}{|k|-\cos \varphi}=\frac{2 \pi}{\sqrt{k^{2}-1}} \times\left[\frac{v}{2 \pi}\right]+\mathcal{O}(1) .
$$

Observe still that the constant energy of these geodesics equals

$$
g_{i j}^{a}\left(\gamma_{t}\right) \frac{d \gamma_{t}^{i}}{d t} \frac{d \gamma_{t}^{j}}{d t}=\left(1+k^{2} a^{2}\right) C^{2}
$$

Hence prescribing speed 1 gives $|C|$ as a function of $|k|$.

We have finally shown the following.

Proposition 2 For any geodesic $\gamma(t)=(y(t), x(t), \theta(t))$ of speed 1 of $G$, there exist constants $C \in[-1,1], C^{\prime}>0$, and $t_{0}, x_{0}, \theta_{0} \in \mathbb{R}$ such that one of the four following cases occurs. We let $k \in[-1,1]$ satisfy $\left(1+k^{2} a^{2}\right) C^{2}=1$. Case 1 The projection on $\mathbb{H}^{2}$ is a straight line (quasi-geodesic or horocycle), or a point.

Case 2 $|k|=1$. The projection on $\mathbb{H}^{2}$ is the horocycle having equation $\left.\overline{\left(x-x_{0}\right.}\right)^{2}+\left(y-C^{\prime} / 2\right)^{2}=\left(C^{\prime} / 2\right)^{2}$. Precisely, we have

$$
\begin{aligned}
& y(t)=\frac{C^{\prime}}{1+C^{2}\left(t-t_{0}\right)^{2}}, \\
& x(t)=x_{0}+\frac{C C^{\prime}\left(t-t_{0}\right)}{1+C^{2}\left(t-t_{0}\right)^{2}}, \\
& \theta(t)=\theta_{0}+\frac{t}{C}-2 \operatorname{arctg}\left(C\left(t-t_{0}\right)\right) .
\end{aligned}
$$


Case $3|k|<1$. The projection on $\mathbb{H}^{2}$ is the quasi-geodesic having equation $\left(x-x_{0}\right)^{2}+\left(y+\frac{C^{\prime} k}{1-k^{2}}\right)^{2}=\left(\frac{C^{\prime}}{1-k^{2}}\right)^{2}$. Precisely, we have

$$
\begin{aligned}
& y(t)=\frac{C^{\prime}}{\operatorname{ch}\left[C \sqrt{1-k^{2}}\left(t-t_{0}\right)\right]-k}, \\
& x(t)=x_{0}+\frac{C^{\prime}}{\sqrt{1-k^{2}}} \times \frac{\operatorname{sh}\left[C \sqrt{1-k^{2}}\left(t-t_{0}\right)\right]}{\operatorname{ch}\left[C \sqrt{1-k^{2}}\left(t-t_{0}\right)\right]-k}, \\
& \theta(t)=\theta_{0}+C k a^{2} t-2 \operatorname{arctg}\left(\operatorname{th}\left[C \sqrt{1-k^{2}}\left(t-t_{0}\right) / 2\right]\right) .
\end{aligned}
$$

Case $4 \quad k>1$. The projection on $\mathbb{H}^{2}$ is the circle (totally included in $\mathbb{H}^{2}$ ) having equation $\left(x-x_{0}\right)^{2}+\left(y-\frac{C^{\prime} k}{k^{2}-1}\right)^{2}=\left(\frac{C^{\prime}}{k^{2}-1}\right)^{2}$. Precisely, we have

$$
\begin{aligned}
& y(t)=\frac{C^{\prime}}{k-\cos \left[C \sqrt{k^{2}-1}\left(t-t_{0}\right)\right]}, \\
& x(t)=x_{0}+\frac{C^{\prime}}{\sqrt{k^{2}-1}} \times \frac{\sin \left[C \sqrt{k^{2}-1}\left(t-t_{0}\right)\right]}{k-\cos \left[C \sqrt{k^{2}-1}\left(t-t_{0}\right)\right]}, \\
& \theta(t)=\theta_{0}+C k a^{2} t-\sqrt{k^{2}-1} \int_{0}^{C \sqrt{k^{2}-1} t} \frac{d \varphi}{k-\cos \varphi}=\left(k a^{2}-\sqrt{k^{2}-1}\right) C t+\mathcal{O}(1) .
\end{aligned}
$$

In this case the geodesic has periodic projection, and even is periodic (with Riemannian length $\frac{2 \pi q}{|C| \sqrt{k^{2}-1}}$ ) if $\frac{k a^{2}}{C \sqrt{k^{2}-1}}$ is rational (equal to $p / q$ with $p, q$ relatively prime integers).

In all cases, we have $x^{\prime}(t)^{2}+y^{\prime}(t)^{2}=C^{2} y(t)^{2}$ for any real $t$, so the projection on $\mathbb{H}^{2}$ has constant energy (speed), and it is the intersection of $\mathbb{H}^{2}$ with an Euclidian circle or line.

Corollary 2 Let $\left(\gamma_{t}\right)$ denote the geodesic of $G$ generated by $(y, x, \theta, u, v, w)$ $\in T^{1} G$, where $\gamma_{0}=(y, x, \theta)$ is the base point in Iwasawa coordinates, and $(u, v, w)$ are the coordinates of $\gamma_{0}^{\prime}$ in the basis $\left(y \frac{\partial}{\partial y}, y \frac{\partial}{\partial x}, \frac{\partial}{\partial \theta}\right)$ of $T_{(y, x, \theta)}^{1} G$. So that (using Lemma 4) we have $w=-v \pm a \sqrt{1-C^{2}}=: w^{ \pm}(u, v)$, with $C^{2}:=u^{2}+v^{2}$.

Then the geodesic $\left(\gamma_{t}\right)$ lives on the leaf of $T^{1} G$, say $L\left(C^{2},+\right)$, having equations $\left\{u^{2}+v^{2}=C^{2}, w=w^{+}(u, v)\right\}$, or on the leaf $L\left(C^{2},-\right)$, having equations $\left\{u^{2}+v^{2}=C^{2}, w=w^{-}(u, v)\right\}$, and has closed (periodic) projection on $\mathbb{H}^{2}$ if and only if $C^{2}<\frac{1}{1+a^{2}}$.

Remark 4 1) The geodesics of $G$ which project on a geodesic of $\mathbb{H}^{2}$ correspond to $k=0$, or equivalently to $C^{2}=1$, and then are exactly the horizontal geodesics of $G \equiv T^{1} \mathbb{H}^{2}, \mathbb{H}^{2}$ being endowed with its Levi-Civita connexion. 
2) The quasi-geodesic $\widetilde{\gamma}_{t}=(y(t), x(t))$ of case 3 in Proposition 2 above is at bounded distance of the geodesic $g$ of $\mathbb{H}^{2}$ having the same ends:

$$
g_{t}=\left(\frac{C^{\prime}}{\sqrt{1-k^{2}} \operatorname{ch}(C t)}, x_{0}+\frac{C^{\prime} \operatorname{sh}(C t)}{\sqrt{1-k^{2}} \operatorname{ch}(C t)}\right) .
$$

More precisely, we see by an easy computation that for any real $t$

$$
\operatorname{ch}\left[\operatorname{dist}\left(\widetilde{\gamma}_{t}, g_{\sqrt{1-k^{2}} t}\right)\right]=1 / \sqrt{1-k^{2}}=\operatorname{ch}\left[\operatorname{dist}\left(\widetilde{\gamma}_{t}, g\right)\right]
$$

This is the same (as it must be by changing the point at $\infty$ ) for the quasigeodesics (non-horizontal half-lines) of case 1 in Proposition 2.

Consequently, all quasi-geodesics we get as projections on $\mathbb{H}^{2}$ of the geodesics of $G$ are made of equidistant points with respect to some geodesic of $\mathbb{H}^{2}$.

3) The case 1 in Proposition 2 above appears as exceptional only due to the choice of a particular point of $\partial \mathbb{H}^{2}$ sent to $\infty$ in the Poincaré halfplane model. This choice is directly dependent of the choice of the Iwasawa coordinates on $G$.

\subsection{Exponential geodesics}

Making a stronger use of the Lie group structure of $G=P S L_{2}(\mathbb{R})$, we find among the geodesics which ones are given by exponentials.

Denote by $\nabla$ the Levi-Civita connexion of $G$ (of course still equipped with its Riemannian structure $\left(\left(g_{i j}^{a}\right)\right)$ of Section 5). Again a somewhat tedious but direct computation (note however that by left-invariance it is enough to make this computation at the unit element of $G$; or alternatively to use the formula

$$
\nabla_{\mathcal{L}_{i}} \mathcal{L}_{j}=\frac{1}{2} \sum_{k}\left(c_{i, j}^{k}+\frac{\left\|\mathcal{L}_{j}\right\|^{2}}{\left\|\mathcal{L}_{k}\right\|^{2}} c_{k, i}^{j}-\frac{\left\|\mathcal{L}_{i}\right\|^{2}}{\left\|\mathcal{L}_{k}\right\|^{2}} c_{j, k}^{i}\right) \mathcal{L}_{k}
$$

where the $c_{i, j}^{k}$ are the structure constants of $\mathcal{G}$ ) gives the following covariant derivatives for our basic left-invariant vector fields:

$$
\begin{aligned}
& \nabla_{\mathcal{L}_{\lambda}} \mathcal{L}_{\lambda}=\nabla_{\mathcal{L}_{\alpha}} \mathcal{L}_{\alpha}=\nabla_{\mathcal{L}_{\kappa}} \mathcal{L}_{\kappa}=0 ; \nabla_{\mathcal{L}_{\alpha}} \mathcal{L}_{\lambda}=\frac{1}{2} \mathcal{L}_{\kappa}=-\nabla_{\mathcal{L}_{\lambda}} \mathcal{L}_{\alpha} ; \\
& \nabla_{\mathcal{L}_{\lambda}} \mathcal{L}_{\kappa}=\frac{1}{2 a^{2}} \mathcal{L}_{\alpha} ; \nabla_{\mathcal{L}_{\kappa}} \mathcal{L}_{\lambda}=\left(1+\frac{1}{2 a^{2}}\right) \mathcal{L}_{\alpha} ; \\
& \nabla_{\mathcal{L}_{\alpha}} \mathcal{L}_{\kappa}=\frac{-1}{2 a^{2}} \mathcal{L}_{\lambda} ; \nabla_{\mathcal{L}_{\kappa}} \mathcal{L}_{\alpha}=-\left(1+\frac{1}{2 a^{2}}\right) \mathcal{L}_{\lambda}
\end{aligned}
$$

As a consequence we see that 


$$
\nabla_{\left(u \mathcal{L}_{\lambda}+v \mathcal{L}_{\alpha}+w \mathcal{L}_{\kappa}\right)}\left(u \mathcal{L}_{\lambda}+v \mathcal{L}_{\alpha}+w \mathcal{L}_{\kappa}\right)=\left(1+a^{-2}\right) w\left(u \mathcal{L}_{\alpha}-v \mathcal{L}_{\lambda}\right) .
$$

Hence we get the geodesics of $G$ which are given by exponentials as the oneparameter left cosets generated by the vector fields $a \mathcal{L}_{\kappa}$ and $(\cos \varphi) \mathcal{L}_{\lambda}+$ $(\sin \varphi) \mathcal{L}_{\alpha}, \varphi \in \mathbb{R} / 2 \pi \mathbb{Z}$.

Equivalently, these are the following exponentials:

$$
t \mapsto g_{0} \exp [a t \kappa]=g\left(z_{0}, \theta_{0}+a t / 2\right),
$$

and

$$
\begin{aligned}
& t \mapsto g_{0} \exp [(t \cos \varphi) \lambda+(t \sin \varphi) \alpha] \\
& \quad=g_{0}\left(\begin{array}{cc}
\operatorname{ch}(t / 2)+\sin \varphi \operatorname{sh}(t / 2) & \cos \varphi \operatorname{sh}(t / 2) \\
\cos \varphi \operatorname{sh}(t / 2) & \operatorname{ch}(t / 2)-\sin \varphi \operatorname{sh}(t / 2)
\end{array}\right),
\end{aligned}
$$

which are respectively the vertical and horizontal geodesics.

\section{Ergodic measures for the geodesic flow on $G / \Gamma^{\prime}$}

Corollary 2 above shows that the leaves $u^{2}+v^{2}=C^{2}$ of $T^{1} G / \Gamma^{\prime}$ are stable under the geodesic flow, disjoint, and that they are made of closed geodesics for $C^{2}<\frac{1}{1+a^{2}}$. Hence we have the following necessary condition for an ergodic measure to exist.

Corollary 3 Any ergodic invariant measure for the geodesic flow on $G / \Gamma^{\prime}$ must be carried by a leaf $L\left(C^{2}, \varepsilon\right)$ of equation $\left\{u^{2}+v^{2}=C^{2}, w=w^{\varepsilon}(u, v)\right\}$ (in the basis chosen in Corollary 2 above, with $C^{2} \in[0,1]$ and $\varepsilon= \pm$ ). Moreover if $C^{2}<\frac{1}{1+a^{2}}$ and if $\frac{k a^{2}}{C \sqrt{k^{2}-1}}$ is rational, then it must be carried by some closed (periodic) geodesic.

Notice that the ergodic invariant measures for the geodesic flow on $G / \Gamma^{\prime}$ which are carried by a leaf $L\left(C^{2}, \varepsilon\right)$ such that $C^{2}<\frac{1}{1+a^{2}}$ would lead to a more or less trivial asymptotic result for the integrals of harmonic 1-forms along the geodesic flow on $G / \Gamma^{\prime}$. So we drop them henceforth.

Lemma 6 For $\left(C^{2}, \varepsilon\right)$ fixed such that $1 \geq C^{2}>\frac{1}{1+a^{2}}$, there is a natural one-to-one map $\psi=\psi_{\varepsilon}^{C}$ from the leaf $L\left(C^{2}, \varepsilon\right)$ (seen as made of geodesics of $G / \Gamma^{\prime}$ having initial values $\theta_{0}=0$ for their angular part $\theta_{t}$ ) onto the set of geodesics of $\mathbb{H}^{2} / \Gamma^{\prime}$. This map goes as follows: with any geodesic $\gamma$ of the leaf $L\left(C^{2}, \varepsilon\right)$, associate successively the projection $\widetilde{\gamma}$ on $\mathbb{H}^{2}$ of its lift to $G$, and the projection $\psi(\gamma)$ on $\mathbb{H}^{2} / \Gamma^{\prime}$ of the geodesic of $\mathbb{H}^{2}$ at bounded distance of $\widetilde{\gamma}$.

This map makes sense as well between the set of line-elements of $L\left(C^{2}, \varepsilon\right)$ and $T^{1}\left(\mathbb{H}^{2} / \Gamma^{\prime}\right)$. 
Proof. Proposition 2 (case 3), Corollary 2 and Remark $(4,2)$ insure that our map $\psi=\psi_{\varepsilon}^{C}$ is well defined. Note indeed the necessary $\Gamma^{\prime}$-invariance: if two line-elements in $T^{1} G$ can be identified modulo some $g^{\prime} \in \Gamma^{\prime}$, then the same $g^{\prime}$ identifies their projections in $\mathbb{H}^{2} / \Gamma^{\prime}$. In the reverse sense, to any geodesic $\psi(\gamma)$ of $\mathbb{H}^{2} / \Gamma^{\prime}$ corresponds a unique geodesic lifted to $\mathbb{H}^{2}$, then two quasi-geodesics in $\mathbb{H}^{2}$ at constant distance

$$
\frac{1}{\sqrt{1-k^{2}}}=\frac{a}{\sqrt{a^{2}+1-C^{-2}}}
$$

of this lift, according to the sign of $k$. Then since the formulas of Case 3 in Proposition 2 give

$$
\theta^{\prime}(t)+\frac{x^{\prime}(t)}{y(t)}=C a^{2} k= \pm a \sqrt{1-C^{2}},
$$

we see that the choice of $\varepsilon$ prescribes the sign of $k$ (the sign of $C$ determining the sense of the geodesic $\psi(g)$ ), and then a unique quasi-geodesic, whence by the equations for the geodesics of $G$ a unique $\gamma$ (for any prescribed initial value $\theta_{0}$ of the angular part). By using furthermore the orthogonal projection in $\mathbb{H}^{2}$ between our quasi-geodesics and their associated geodesic, we get at once the analogous map at the level of line-elements.

Remark 5 Note that in fact each leaf $L\left(C^{2}, \varepsilon\right)$ splits into a continuum of sub-leaves:

$$
L\left(C^{2}, \varepsilon\right)=\bigcup_{\theta_{0} \in \mathbb{R} / 2 \pi \mathbb{Z}} L\left(C^{2}, \varepsilon, \theta_{0}\right),
$$

taking into account the initial value $\theta_{0}$ of the angular part (either at time 0 , or above the orthogonal projection of the fixed point $\sqrt{-1}$ on the quasigeodesic $\widetilde{\gamma}$ ) of the geodesic $\gamma$. Thus this is indeed the set of its line-elements of each sub-leaf $L\left(C^{2}, \varepsilon, \theta_{0}\right)$, which is set in one-to-one correspondence with $T^{1}\left(\mathbb{H}^{2} / \Gamma^{\prime}\right) \equiv G / \Gamma^{\prime}$ by the map $\psi=\psi_{\varepsilon, \theta_{0}}^{C}$. Note that $L\left(C^{2}, \varepsilon, \theta_{0}\right)$ has indeed 3 dimensions, as $G$. However, this initial value $\theta_{0}$ will not matter anyway in the following, so that we drop it henceforth, going on with the shorter notation $L\left(C^{2}, \varepsilon\right), \psi_{\varepsilon}^{C}$.

Now it is known (see [8]) that the Liouville measure on $T^{1}\left(\mathbb{H}^{2} / \Gamma^{\prime}\right)$ is invariant and ergodic under the geodesic flow. This fact and Lemma 6 above allow therefore the following.

Definition 1 For $\left(C^{2}, \varepsilon\right)$ fixed such that $1 \geq C^{2}>\frac{1}{1+a^{2}}$, denote by $\mu_{\varepsilon}^{C}$ the image of the normalized Liouville measure $\mu$ on $T^{1}\left(\mathbb{H}^{2} / \Gamma^{\prime}\right) \equiv G / \Gamma^{\prime}$ under the map $\psi_{\varepsilon}^{C}$ of Lemma 6. So $\mu_{\varepsilon}^{C}$ is a probability measure on the set of line-elements of the leaf $L\left(C^{2}, \varepsilon\right)$, which is invariant and ergodic under the geodesic flow on $G / \Gamma^{\prime}$. 


\section{Asymptotic geodesic windings}

We fix here a leaf $L\left(C^{2}, \varepsilon\right)$, and endow it with the ergodic invariant probability measure $\mu_{\varepsilon}^{C}$ of Definition 1 . We want to obtain the asymptotic law under $\mu_{\varepsilon}^{C}$ of

$$
\left(t^{-1} \int_{\gamma[0, t]} \omega_{0}, t^{-1 / 2} \int_{\gamma[0, t]} \omega_{1}, t^{-1 / 2} \int_{\gamma[0, t]} \omega_{2}\right) \quad \text { as } \quad t \rightarrow \infty,
$$

where the geodesic $\gamma$ of $G / \Gamma^{\prime}$ is chosen (at time 0 ) according to $\mu_{\varepsilon}^{C}$ and $\gamma[0, t]$ denotes this geodesic $\gamma$ run during the time-interval $[0, t]$.

Note that by the $\Gamma^{\prime}$-invariance of the forms $\omega_{j}$ it makes no difference to think of the geodesics $\gamma$ as started in the fundamental domain $D$ and living on $G$, the forms being harmonic on $G$ as well.

The following lemma reduces essentially our study of the geodesics of $G$ to a study of the geodesics of $\mathbb{H}^{2}$.

Lemma $7 \quad$ As $t \rightarrow \infty$, the asymptotic law of

$$
\left(t^{-1} \int_{\gamma[0, t]} \omega_{0}, t^{-1 / 2} \int_{\gamma[0, t]} \omega_{1}, t^{-1 / 2} \int_{\gamma[0, t]} \omega_{2}\right)
$$

under $\mu_{\varepsilon}^{C}=\mu_{\varepsilon}^{C}(d \gamma)$ is the same as the asymptotic law under the Liouville measure $\mu=\mu(d g)$ on $T^{1}\left(\mathbb{H}^{2} / \Gamma^{\prime}\right)$ of the following ( $\omega_{0}^{\prime}$ denoting $\left.\left(\omega_{0}-d \theta\right)\right)$ :

$$
\begin{aligned}
& \left(\varepsilon a \sqrt{1-C^{2}}+\left(\frac{a t}{\sqrt{1-C^{2}}}\right)^{-1} \int_{g[0, t]} \omega_{0}^{\prime},\right. \\
& \left.\left(\frac{a t}{\sqrt{1-C^{2}}}\right)^{-1 / 2} \int_{g[0, t]} \omega_{1},\left(\frac{a t}{\sqrt{1-C^{2}}}\right)^{-1 / 2} \int_{g[0, t]} \omega_{2}\right) .
\end{aligned}
$$

Proof. Let us deal first with $\omega_{1}, \omega_{2}$. By Definition 1 and Lemma 6 and the fact that $\omega_{j}=\pi^{*} \omega_{j}$ for $1 \leq j \leq 2, \pi$ denoting here the canonical projection from $G \equiv T^{1} \mathbb{H}^{2}$ onto $\mathbb{H}^{2}$, we just have to compare

$$
t^{-1 / 2} \int_{\gamma[0, t]} \omega_{j}=t^{-1 / 2} \int_{\widetilde{\gamma}[0, t]} \omega_{j} \quad \text { with } \quad t^{-1 / 2} \int_{\psi(\gamma)[0, t]} \omega_{j} .
$$

Now use that on $\mathbb{H}^{2} \omega_{j}=d F_{j}$ is exact, and recall from Remark $(4,2)$ that the geodesic $\psi(\gamma)$ must be run at speed $|C| \sqrt{1-k^{2}}=\sqrt{1-C^{2}} / a$, to get:

$$
\begin{aligned}
\left|\int_{\widetilde{\gamma}[0, t]} \omega_{j}-\int_{\psi(\gamma)\left[0, \sqrt{1-C^{2}} \frac{t}{a}\right]} \omega_{j}\right|= & \mid F_{j}(\widetilde{\gamma}(t))-F_{j}\left(\psi(\gamma)\left(\sqrt{1-C^{2}} \frac{t}{a}\right)\right) \\
& -F_{j}(\widetilde{\gamma}(0))+F_{j}(\psi(\gamma)(0)) \mid \\
\leq & 2\left\|\omega_{j}\right\|_{\infty} / \sqrt{1-k^{2}} .
\end{aligned}
$$


This shows that

$$
t^{-1 / 2}\left(\int_{\widetilde{\gamma}[0, t]} \omega_{j}-\int_{\psi(\gamma)\left[0, \sqrt{1-C^{2}} \frac{t}{a}\right]} \omega_{j}\right)
$$

goes uniformly to 0 , whence the result relating to $\omega_{1}, \omega_{2}$.

Now we have to deal with $\omega_{0}$, which from Theorem 1 writes $\omega_{0}=d \theta+\omega_{0}^{\prime}$, with $\omega_{0}^{\prime}=\pi^{*} \omega_{0}^{\prime}$. Thus we can handle $\omega_{0}^{\prime}$ as $\omega_{1}, \omega_{2}$ above, to get:

$\int_{\gamma[0, t]} \omega_{0}^{\prime}=\int_{\widetilde{\gamma}[0, t]} \omega_{0}^{\prime}=\int_{\psi(\gamma)\left[0, \sqrt{1-C^{2}} \frac{t}{a}\right]} \omega_{0}^{\prime}+\mathcal{O}(1) \times(\tilde{Y}(\psi \circ \gamma, 0)+\tilde{Y}(\psi \circ \gamma, t))$,

where

$$
\tilde{Y}(g, t):=\sup \left\{\tilde{y}(z) \mid \operatorname{dist}\left(g\left(\sqrt{1-C^{2}} \frac{t}{a}\right), z\right) \leq \frac{1}{\sqrt{1-k^{2}}}\right\} .
$$

On the other hand we have by Proposition 2 (Case 3) and the proof of Lemma 6:

$$
\int_{\gamma[0, t]} d \theta=C k a^{2} t+\mathcal{O}(1)=\varepsilon a \sqrt{1-C^{2}} t+\mathcal{O}(1)
$$

Therefore the asymptotic law of $t^{-1} \int_{\gamma[0, t]} \omega_{0}$ under $\mu_{\varepsilon}^{C}(d \gamma)$ is the same as the asymptotic law of

$$
\varepsilon a \sqrt{1-C^{2}}+t^{-1} \int_{g\left[0, \sqrt{1-C^{2}} \frac{t}{a}\right]} \omega_{0}^{\prime}+\mathcal{O}(\tilde{Y}(g, 0)+\tilde{Y}(g, t)) / t
$$

under $\mu(d g)$.

Observe further that under $\mu(d g)$ the process $\tilde{Y}(g, t)$ is stationary, so that the last term above asymptotically vanishes in probability. Hence we have shown that the asymptotic law of $t^{-1} \int_{\gamma[0, t]} \omega_{0}$ under $\mu_{\varepsilon}^{C}(d \gamma)$ is the same as the asymptotic law of

$$
\varepsilon a \sqrt{1-C^{2}}+t^{-1} \int_{g\left[0, \sqrt{1-C^{2}} \frac{t}{a}\right]} \omega_{0}^{\prime}
$$

under $\mu(d g)$.

Finally the result is valid jointly for the term with $\omega_{0}$ and the two others, since for each the neglected contributions vanish in probability. It remains only to replace $t$ by $\frac{a t}{\sqrt{1-C^{2}}}$.

The following theorem describes the asymptotic geodesic windings in $G / \Gamma^{\prime}$, under the ergodic measures of Section 12 . 
Theorem 3 Let us consider a fixed leaf $L\left(C^{2}, \varepsilon\right)$ (defined in Corollary 3) of $G / \Gamma^{\prime}$, with $1 \geq C^{2}>\frac{1}{1+a^{2}}$, endowed with the ergodic invariant probability measure $\mu_{\varepsilon}^{C}$ of Definition 1.

Then the law under $\mu_{\varepsilon}^{C}=\mu_{\varepsilon}^{C}(d \gamma)$ of

$$
\left(t^{-1} \int_{\gamma[0, t]} \omega_{0}, t^{-1 / 2} \int_{\gamma[0, t]} \omega_{1}, t^{-1 / 2} \int_{\gamma[0, t]} \omega_{2}\right)
$$

converges as $t \rightarrow \infty$ to the law of

$$
\left(\varepsilon a \sqrt{1-C^{2}}+\left(\frac{2 \sqrt{1-C^{2}}}{a}\right) \mathcal{C},\left(\frac{2 \sqrt{1-C^{2}}}{a}\right)^{1 / 2} \mathcal{N}^{1},\left(\frac{2 \sqrt{1-C^{2}}}{a}\right)^{1 / 2} \mathcal{N}^{2}\right)
$$

where the variables $\mathcal{C}, \mathcal{N}^{1}, \mathcal{N}^{2}$ are independent, $\mathcal{C}$ is Cauchy with parameter $\frac{1}{2}$, and $\mathcal{N}^{1}, \mathcal{N}^{2}$ are centred Gaussian with variance $\frac{3}{\pi} \int_{D_{0}}|\eta(z)|^{8} d x d y$.

Note a clear difference between the Brownian and geodesic behaviors: mainly, here (counter to the Brownian case) the $d \theta$-part of the form $\omega_{0}$ is responsible for a non-negligible asymptotic contribution. Moreover the parameter $a$ now appears in the limit law.

This makes a noteworthy contrast with the hyperbolic case (see [2], [3], [6]).

This difference appears in Lemma 7 above, whereas once the $d \theta$-part has been moved away, the remaining asymptotic law is essentially the same as the Brownian one, given by Theorem 2. So that our remaining task will be below mainly to compare on $\mathbb{H}^{2}$ the geodesic paths to the Brownian paths, somewhat in the spirit of the methods already employed in $[2,4,6,12]$, but in a more synthetic and simple way, taking advantage of the fact that we have here, somewhat as in [11], to deal only with the closed forms $\omega_{0}, \omega_{0}^{\prime}, \omega_{1}, \omega_{2}$.

\section{Proof of Theorem 3}

The strategy for this proof is mainly to replace the geodesic paths by the Brownian paths, as in [4], [6], [11], in order to reduce Theorem 3 to Theorem 2. But we shall here take advantage of the closedness of our forms $\omega_{0}, \omega_{0}^{\prime}, \omega_{1}, \omega_{2}$, somewhat as in [11], to simplify sensibly the proofs of these articles. In particular, we do not any more have to use a spectral gap, nor to rise to the stable foliation. Another change (and hopefully clarification) with respect to these proofs is the use of a simultaneous disintegration of the Liouville and the Wiener measures: we avowedly condition the Brownian motion (starting from a given point $z \in \mathbb{H}^{2}$ ) to exit the hyperbolic plane at the same point as a given geodesic (starting also from $z$ ). This point of view was more or less implicit in the preceding proofs, but did not appear transparently. 
The asymptotic law we are looking for is given by the asymptotic behavior, as $t \rightarrow \infty$ and for $\ell \in \mathbb{R}^{3}$, of the following quantity:

$$
J_{t}^{\ell}:=\int_{G / \Gamma^{\prime}} \exp \left[\sqrt{-1}\left(\frac{\ell_{0}}{t} \int_{g[0, t]} \omega_{0}^{\prime}+\frac{\ell_{1}}{\sqrt{t}} \int_{g[0, t]} \omega_{1}+\frac{\ell_{2}}{\sqrt{t}} \int_{g[0, t]} \omega_{2}\right)\right] \mu(d g) .
$$

\subsection{Conditionning by the end-points}

Using the notation $g=g(z, \theta)=g(y, x, \theta)$ and the expression of $\mu=$ $\mu(y, x, \theta)$ in the Iwasawa coordinates (seen in Section 5), and setting $\omega_{0}^{\prime \prime}:=$ $\ell_{0} \omega_{0}^{\prime}, \omega:=\ell_{1} \omega_{1}+\ell_{2} \omega_{2}$, we have

$$
J_{t}^{\ell}=\int_{D} \int_{0}^{2 \pi} \exp \left[\sqrt{-1}\left(t^{-1} \int_{g(y, x, \theta)[0, t]} \omega_{0}^{\prime \prime}+t^{-1 / 2} \int_{g(y, x, \theta)[0, t]} \omega\right)\right] d \theta \frac{d x d y}{4 \pi^{2} y^{2}}
$$

Then for $(z=x+\sqrt{-1} y, \theta) \in \mathbb{H}^{2} \times(\mathbb{R} / 2 \pi \mathbb{Z})$, denote by $\left(z_{t}^{\theta}\right)$ the geodesic defined by $g(z, \theta)$, and by $\mathbb{P}_{z}^{\theta}$ the law of the Brownian motion $\left(Z_{t}^{\theta}\right)$ of $\mathbb{H}^{2}$, started from $z$ and conditioned to exit $\mathbb{H}^{2}$ at the positive end $z_{\infty}^{\theta}$ of the geodesic of $\mathbb{H}^{2}$ defined by $g(z, \theta)$.

Consider then the the hitting time by the coordinate process $\left(Z_{t}\right)$ of the stable horocycle defined by $\left(z_{\infty}^{\theta}, z_{t}^{\theta}\right)$, say $h_{t}$. It is defined precisely by

$$
h_{t}=h_{t}^{z, \theta}:=\inf \left\{s>0 \mid B_{z_{\infty}^{\theta}}\left(z, Z_{s}\right)=e^{t}\right\},
$$

where

$$
\left(z, z^{\prime}\right) \mapsto B_{u}\left(z, z^{\prime}\right)=p\left(z^{\prime}, u\right) / p(z, u)
$$

denotes the Busemann function based at $u \in \partial \mathbb{H}^{2}, \quad p$ denoting the Poisson kernel.

The following lemma insures that the disintegration of the Liouville and Wiener measures is simultaneous, by conditioning with respect to the endpoint $z_{\infty}^{\theta}$. A reason for that is that the harmonic measures at $\partial \mathbb{H}^{2}$ are the same for both, namely $p(z, u) d u$.

\section{Lemma 8}

$$
\mathbb{P}_{z}:=\int_{0}^{2 \pi} \mathbb{P}_{z}^{\theta} \frac{d \theta}{2 \pi}
$$

is the Wiener measure started from $z$, for any $z \in \mathbb{H}^{2} / \Gamma^{\prime}$, and

$$
\mathbb{P}_{\mu}:=\int \mathbb{P}_{z}^{\theta} d \mu(z, \theta)
$$

is the stationary Wiener measure on $\mathbb{H}^{2} / \Gamma^{\prime}$. 
Proof. $\quad\left(Z_{t}^{\theta}\right)$ is by definition the $h$-process of the unconditioned Brownian motion, with $h(z)=p\left(z, z_{\infty}^{\theta}\right), p(z, u)=y /|z-u|^{2}$ still denoting the Poisson kernel.

Hence we have for any $(z, \theta)$, any $t$ and any $\mathcal{F}_{t}$-measurable positive functional $F_{t}$ :

$$
\mathbb{E}_{z}^{\theta}\left[F_{t}\right]=\mathbb{E}_{z}\left[B_{z_{\infty}^{\theta}}\left(z, Z_{t}\right) \times F_{t}\right] .
$$

The first identity of the lemma follows, since for any $z, \theta, Z$ we have

$$
\int_{0}^{2 \pi} B_{z_{\infty}^{\theta}}(z, Z) d \theta=2 \int_{\mathbb{R}} B_{u}(z, Z) p(z, u) d u=2 \int_{\mathbb{R}} p(Z, u) d u=2 \pi .
$$

Integrating this first identity with respect to the normalized volume measure $\frac{d x d y}{2 \pi y^{2}}$ gives immediately the second identity of the lemma.

\subsection{From geodesics to Brownian paths}

We perform here the substitution of the Brownian paths for the geodesics. Our first aim is to establish the following.

Proposition 3 Ast $\rightarrow \infty, J_{t}^{\ell}$ (defined just before Section 14.1) behaves as

$$
K_{t}^{\ell}:=\int_{D} \int_{0}^{2 \pi} \mathbb{E}_{z}^{\theta}\left(\exp \left[\frac{\sqrt{-1}}{t} \int_{z}^{Z_{h_{t}}} \omega_{0}^{\prime \prime}+\frac{\sqrt{-1}}{\sqrt{t}} \int_{z}^{Z_{h_{t}}} \omega\right]\right) d \theta \frac{d x d y}{4 \pi^{2} y^{2}}
$$

Our forms being closed, we have the following expression for $J_{t}^{\ell}$ :

$$
\begin{aligned}
J_{t}^{\ell}=\int_{D} \int_{0}^{2 \pi} \mathbb{E}_{z}^{\theta}(\exp [ & \frac{\sqrt{-1}}{t}\left(\int_{z}^{Z_{h_{t}}} \omega_{0}^{\prime \prime}+\int_{Z_{h_{t}}}^{z_{t}^{\theta}} \omega_{0}^{\prime \prime}\right) \\
& \left.\left.+\frac{\sqrt{-1}}{\sqrt{t}}\left(\int_{z}^{Z_{h_{t}}} \omega+\int_{Z_{h_{t}}}^{z_{t}^{\theta}} \omega\right)\right]\right) d \theta \frac{d x d y}{4 \pi^{2} y^{2}} .
\end{aligned}
$$

Applying the isometry $f_{z, \theta}$ of $\mathbb{H}^{2}$ which maps $g(1,0)$ to $g(z, \theta)$, we see that the law of $\int_{Z_{h_{t}}}^{z_{t}^{\theta}} \omega$ under $\mathbb{P}_{z}^{\theta}$ is the same as the law of

$$
\int_{Z_{h_{t}}^{0}}^{e_{t}} f_{z, \theta}^{*} \omega
$$

where $e_{t}:=\sqrt{-1} e^{t}$ and $Z_{h_{t}}^{0}$ is the point at which the Brownian motion $\left(Z_{t}^{0}\right)$ started from $\sqrt{-1}$ and conditioned to exit at $\infty$ hits the horizontal horocycle having equation $y=e^{t}$. 
Now $\left(Z_{t}^{0}\right)$ is the $h$-process of the unconditioned Brownian motion, with $h(z)=p(z, \infty) \equiv y$, so that its infinitesimal generator is

$$
\frac{1}{2} y^{-1} \Delta \circ y=\frac{1}{2} \Delta+y \partial_{y},
$$

$\Delta$ denoting the Laplacian of $\mathbb{H}^{2}$, and then we have

$$
Z_{t}^{0}=\sqrt{-1} e^{w_{t}+t / 2}+\int_{0}^{t} e^{w_{s}+s / 2} d W_{s},
$$

for two independent standard real Brownian motions $\left(w_{t}\right)$ and $\left(W_{t}\right)$.

As a consequence, using the boundedness of $\omega$, we have

$$
\int_{Z_{h_{t}}^{0}}^{e_{t}} f_{z, \theta}^{*} \omega=\mathcal{O}\left(e^{-t} \times\left|\int_{0}^{\inf \left\{s \mid w_{s}+s / 2=t\right\}} e^{w_{s}+s / 2} d W_{s}\right|\right) .
$$

The technical Brownian behavior we need now and after is given by the following.

Lemma 9 As $t \rightarrow \infty$,

$$
e^{-t} \int_{0}^{\inf \left\{s \mid w_{s}+s / 2=t\right\}} e^{w_{s}+s / 2} d W_{s}
$$

converges in law, and $\inf \left\{s \mid w_{s}+s / 2=t\right\}=2 t+o\left(t^{q}\right)$ almost surely, for any $q \in] 1 / 2,1]$.

Proof. Fix $c \in \mathbb{R}$, and look for a $C^{2}$ function $f$ on $\mathbb{R}_{+}$such that

$$
R_{t}:=e^{-\left(c^{2} / 2\right) \int_{0}^{t}\left(y_{s}^{0}\right)^{2} d s} f\left(y_{t}^{0}\right)
$$

be a martingale, with $y_{t}^{0}=e^{w_{t}+t / 2}$.

$\left(y_{t}^{0}\right)$ having generator $\frac{1}{2} y^{2} \partial_{y}^{2}+y \partial_{y}$, we have by Itô's formula

$$
\begin{aligned}
R_{t}=f(1)+\operatorname{mart}+\frac{1}{2} \int_{0}^{t} e^{-\left(c^{2} / 2\right) \int_{0}^{s}\left(y_{v}^{0}\right)^{2} d v} & \\
& \times\left(y_{s}^{0}\right)^{2} \times\left[f^{\prime \prime}\left(y_{s}^{0}\right)+2\left(y_{s}^{0}\right)^{-1} f^{\prime}\left(y_{s}^{0}\right)-c^{2} f\left(y_{s}^{0}\right)\right] d s,
\end{aligned}
$$

whence the equation:

$$
f^{\prime \prime}(y)+2 y^{-1} f^{\prime}(y)-c^{2} f(y)=0 .
$$

Setting $f_{1}(y):=\sqrt{y} f(y)$, this gives

$$
f_{1}^{\prime \prime}(y)+y^{-1} f_{1}^{\prime}(y)-\left(c^{2}+(2 y)^{-2}\right) f_{1}(y)=0 .
$$


Since $f_{1}$ must be bounded near 0 , we have, up to some multiplicative constant:

$$
f(y)=(c y)^{-1 / 2} I_{1 / 2}(c y)=\sum_{k \geq 0} \frac{(c y)^{2 k}}{2^{2 k+\frac{1}{2}} k ! \Gamma\left(2 k+\frac{3}{2}\right)},
$$

where $I_{r}$ denotes the usual modified Bessel function.

The optional sampling theorem then gives

$$
\begin{aligned}
\mathbb{E}\left[\exp \left(\sqrt{-1} c \int_{0}^{\inf \left\{s \mid w_{s}+s / 2=t\right\}} e^{w_{s}+s / 2} d W_{s}\right)\right] \\
\quad=\mathbb{E}\left[\exp \left(-\frac{c^{2}}{2} \int_{0}^{\inf \left\{s \mid y_{s}^{0}=e^{t}\right\}}\left(y_{s}^{0}\right)^{2} d s\right)\right]=\frac{f(1)}{f\left(e^{t}\right)} .
\end{aligned}
$$

Changing $c$ into $c e^{-t}$, we get as $t \rightarrow \infty$ :

$$
\begin{aligned}
\mathbb{E}\left[\exp \left(\sqrt{-1} c e^{-t} \int_{0}^{\inf \left\{s \mid w_{s}+s / 2=t\right\}} e^{w_{s}+s / 2} d W_{s}\right]\right. \\
\longrightarrow\left(\sum_{k \geq 0} \frac{\Gamma(3 / 2) c^{2 k}}{2^{2 k} k ! \Gamma\left(2 k+\frac{3}{2}\right)}\right)^{-1} \in L^{2}(\mathbb{R}, d c),
\end{aligned}
$$

which proves the first sentence of the lemma.

Finally, the second sentence of the lemma is straightforward from the following observation: setting again

$$
h_{t}=h_{t}^{\sqrt{-1}, 0}=\inf \left\{s \mid w_{s}+s / 2=t\right\}=\inf \left\{s \mid y_{s}^{0}=e^{t}\right\},
$$

we have $t=\log y_{h_{t}}^{0}=\frac{1}{2} h_{t}+w_{h_{t}}=\frac{1}{2} h_{t}+o\left(\left(h_{t}\right)^{q}\right)$.

As a consequence of this lemma and of the above, we see that

$$
t^{-1 / 2} \int_{Z_{h_{t}}}^{z_{t}^{\theta}} \omega
$$

goes to 0 in $\mathbb{P}_{z}^{\theta}$-probability. This proves half of Proposition 3.

We have now to deal with the law of

$$
t^{-1} \int_{Z_{h_{t}}}^{z_{t}^{\theta}} \omega_{0}^{\prime \prime}
$$

under $\mathbb{P}_{z}^{\theta}$, or equivalently by the same reason as above for $\omega$, with the law of

$$
t^{-1} \int_{Z_{h_{t}}^{0}}^{e_{t}} f_{z, \theta}^{*} \omega_{0}^{\prime \prime}
$$


This cannot be further handled as above, since the form $\omega_{0}^{\prime \prime}$ is not bounded, whereas $\omega$ was. We only have now the following estimate, by integrating along the horizontal horocycle $y=e^{t}$ containing $e_{t}, Z_{h_{t}}^{\theta}$ :

$$
\begin{aligned}
\left|\int_{Z_{h_{t}}^{0}}^{e_{t}} f_{z, \theta}^{*} \omega_{0}^{\prime}\right| \leq \mid e^{-t} & \int_{0}^{h_{t}} e^{w_{s}+s / 2} d W_{s} \mid \\
& \times \sup \left\{\left|f_{z, \theta}^{*} \omega_{0}^{\prime}\right|_{(\sqrt{-1}+x) e^{t}}|| x|\leq| e^{-t} \int_{0}^{h_{t}} e^{w_{s}+s / 2} d W_{s} \mid\right\},
\end{aligned}
$$

where again $h_{t}=h_{t}^{\sqrt{-1}, 0}=\inf \left\{s \mid y_{s}^{0}=e^{t}\right\}=\inf \left\{s \mid w_{s}+s / 2=t\right\}$.

Fix any $r>0$. Lemma 9 shows that the laws of

$$
e^{-t} \int_{0}^{h_{t}} e^{w_{s}+s / 2} d W_{s}
$$

$t$ large, are tight, and then provides some $R>0$ such that

$$
\mathbb{P}\left[\left|e^{-t} \int_{0}^{h_{t}} e^{w_{s}+s / 2} d W_{s}\right|>R\right]<r
$$

for any large enough positive $t$.

We deduce from these last two estimates that

$$
\begin{aligned}
\mathbb{P}_{z}^{\theta}\left[\left|t^{-1} \int_{Z_{h_{t}}}^{z_{t}^{\theta}} \omega_{0}^{\prime}\right|>r\right] & =\mathbb{P}\left[\left|t^{-1} \int_{Z_{h_{t}}^{0}}^{e_{t}} f_{z, \theta}^{*} \omega_{0}^{\prime}\right|>r\right] \\
& \leq r+1\left\{t^{-1} \sup \left\{\left|f_{z, \theta}^{*} \omega_{0}^{\prime}\right|_{(\sqrt{-1}+x) e^{t}}|| x \mid \leq R\right\}>r / R\right\}
\end{aligned}
$$

and then by integrating against $\mu$ and using Lemma 8 :

$$
\begin{aligned}
\mathbb{P}_{\mu}\left[\left|t^{-1} \int_{Z_{h_{t}}}^{z_{t}^{\theta}} \omega_{0}^{\prime}\right|>r\right] \leq & r+\mu\left[t^{-1} \sup \left\{\left|\omega_{0}^{\prime}\right|_{H_{x}\left(z_{t}^{\theta}\right)}|| x \mid \leq R\right\}>r / R\right] \\
& =r+\mu\left[t^{-1} \sup \left\{\left|\omega_{0}^{\prime}\right|_{H_{x}(z)}|| x \mid \leq R\right\}>r / R\right],
\end{aligned}
$$

where $\left(H_{x}, x \in \mathbb{R}\right)$ denotes the positive horocycle flow. For the last equality, we used the invariance of the Liouville measure $\mu$ under the geodesic flow.

By continuity of $\left|\omega_{0}^{\prime}\right|, \sup \left\{\left|\omega_{0}^{\prime}\right|_{H_{x}(z)}|| x \mid \leq R\right\}$ is finite for every $z$, and thus we just proved:

$$
\mathbb{P}_{\mu}\left[\left|t^{-1} \int_{Z_{h_{t}}}^{z_{t}^{\theta}} \omega_{0}^{\prime}\right|>r\right] \leq 2 r \quad \text { for large enough } t .
$$

Since in the last expression above for $J_{t}^{\ell}$ (immediately after Proposition 3), we were not only under the law $\mathbb{P}_{z}^{\theta}$, but indeed under the law $\mathbb{P}_{\mu}=\int \mathbb{P}_{z}^{\theta} d \mu(z, \theta)$, we have so far proved Proposition 3 . 


\subsection{End of the proof of Theorem 3}

Section 7 allows to denote also by $\mathbb{P}_{\mu}$ the stationary Wiener measure on $G / \Gamma^{\prime}$, since the Brownian motion of $G$ projects on the Brownian motion of $\mathbb{H}^{2}$ (and similarly for the volume measures). Let us recall also that our forms $\omega_{0}^{\prime}, \omega_{1}, \omega_{2}$ come from $\mathbb{H}^{2} / \Gamma^{\prime}$ : they are defined on $G / \Gamma^{\prime}$ and on $\mathbb{H}^{2} / \Gamma^{\prime}$ as well, in other words are invariant under pull back $\pi^{*}$ by the canonical projection. Hence the joint laws of their integrals along the Brownian paths are the same, no matter whether they are understood on $G / \Gamma^{\prime}$ or on $\mathbb{H}^{2} / \Gamma^{\prime}$.

Moreover we have seen in Section 7 also that the angular Brownian component $\theta_{s}$ is a mere one-dimensional Brownian motion. As a consequence, it is immediate that $t^{-1} \int_{g[0, t]} d \theta=\left(\theta_{t}-\theta_{0}\right) / t$ goes to $0 \mathbb{P}_{\mu}$-almost surely. Therefore we can replace in Theorem 2 the form $\omega_{0}$ by the form $\omega_{0}^{\prime}=\omega_{0}-d \theta$.

These remarks show that the following is simply a second version of Theorem 2 (with the notations of Section 14.1 and of Theorem 2).

Corollary 4 We have for any $\ell \in \mathbb{R}^{3}$ :

$$
\begin{aligned}
\lim _{t \rightarrow \infty} \mathbb{E}_{\mu}\left(\operatorname { e x p } \left[\frac{\sqrt{-1}}{t} \int_{Z[0, t]} \omega_{0}^{\prime \prime}\right.\right. & \left.\left.+\frac{\sqrt{-1}}{\sqrt{t}} \int_{Z[0, t]} \omega\right]\right) \\
& =\mathbb{E}\left(\exp \left[\sqrt{-1}\left(\ell_{0} \mathcal{C}+\ell_{1} \mathcal{N}^{1}+\ell_{2} \mathcal{N}^{2}\right)\right]\right)
\end{aligned}
$$

Now Lemma 9 asserts that the time-change $h_{t}=h_{t}^{z, \theta}$ appearing in $K_{t}^{\ell}$ of Proposition 3 satisfies $\quad h_{t}=2 t+o(t) \mathbb{P}_{z}^{\theta}$-almost surely, uniformly with respect to $(z, \theta)$. Indeed, the law under $\mathbb{P}_{z}^{\theta}$ of this $h_{t}$ equals the law of the $h_{t}=h_{t}^{\sqrt{-1}, 0}$ in Lemma 9. So that with arbitrary large probability we can write $h_{t}=2 t+o(t)$ with a uniform deterministic $o(t)$.

This allows to replace $t$ by $h_{t}$ in the formula of Corollary 4 above, and likewise to insure that

$$
\limsup _{t \rightarrow \infty}\left|\mathbb{E}_{\mu}\left(\exp \left[\frac{\sqrt{-1}}{t} \int_{Z[0, t]} \omega_{0}^{\prime \prime}+\frac{\sqrt{-1}}{\sqrt{t}} \int_{Z[0, t]} \omega\right]\right)-K_{t}^{\ell^{\prime}}\right|=0,
$$

with $\ell^{\prime}:=\left(\ell_{0} / 2, \ell_{1} / \sqrt{2}, \ell_{2} / \sqrt{2}\right)$. Therefore using Corollary 4 and Proposition 3 we have proved that

$$
\lim _{t \rightarrow \infty} J_{t}^{\ell}=\mathbb{E}\left(\exp \left[\sqrt{-1}\left(2 \ell_{0} \mathcal{C}+\sqrt{2} \ell_{1} \mathcal{N}^{1}+\sqrt{2} \ell_{2} \mathcal{N}^{2}\right)\right]\right) .
$$

This concludes the proof, since by Lemma 7 and by the very definition of $J_{t}^{\ell}$ (just before Section 14.1) this formula is equivalent to Theorem 3. 


\section{References}

[1] Ayoub, R.: An introduction to the analytic theory of numbers. Mathematical Surveys 10. American Mathematical Society, Providence, R.I., 1963.

[2] Enriquez, N. , Franchi, J. and Le Jan, Y.: Stable windings on hyperbolic surfaces. Probab. Theory Related Fields 119 (2001), no. 2, 213-255.

[3] Enriquez, N. , Franchi, J. and Le Jan, Y.: Central limit theorem for the geodesic flow associated with a Kleinian group, case $\delta>d / 2$. J. Math. Pures Appl. (9) 80 (2001), no. 2, 153-175.

[4] EnRiquez, N. And Le Jan, Y.: Statistic of the winding of geodesics on a Riemann surface with finite area and constant negative curvature. Rev. Mat. Iberoamericana 13 (1997), no. 2, 377-401.

[5] Franchi, J.: Asymptotic singular windings of ergodic diffusions. Stochastic Process. Appl. 62 (1996), 277-298.

[6] Franchi, J.: Asymptotic singular homology of a complete hyperbolic 3manifold of finite volume. Proc. London Math. Soc. (3) 79 (1999), 451-480.

[7] Guivarc'H, Y. and Le Jan Y.: Asymptotic winding of the geodesic flow on modular surfaces and continued fractions. Ann. Sci. École Norm. Sup. (4) 26 (1993), 23-50.

[8] Hopf, E.: Ergodic theory and the geodesic flow on surfaces of constant negative curvature. Bull. Amer. Math. Soc. 77 (1971), 863-877.

[9] Hubbard, J. and Pourezza, I.: The space of closed subgroups of $\mathbb{R}^{2}$. Topology 18 (1979), 143-146.

[10] Ikeda, N. and Watanabe, S.: Stochastic differential equations and diffusion processes. North-Holland, Kodansha, 1981.

[11] Le JAN, Y.: Sur l'enroulement géodésique des surfaces de Riemann. C. R. Acad. Sci. Paris Sér. I Math. 314 (1992), 763-765.

[12] LE JAN, Y.: The central limit theorem for the geodesic flow on noncompact manifolds of constant negative curvature. Duke Math. J. 74 (1994), no. 1, $159-175$.

[13] Milnor, J.: Introduction to algebraic K-theory. Annals of Mathematics Studies 72. Princeton University Press, Princeton, N.J.; University of Tokyo Press, Tokyo, 1971.

[14] Rolfsen, D.: Knots and links. Mathematics Lecture Series 7. Publish or Perish, Berkeley, Calif., 1976.

[15] Revuz, D. And Yor, M.: Continuous martingales and Brownian motion. Third edition. Grundlehren der Mathematischen Wissenschaften 293. Springer-Verlag, Berlin, 1999.

[16] De Sam Lazaro, J. And Meyer, P.A.: Questions de théorie des flots. Lecture Notes in Math. 465, Sém. Probab. IX. Springer, Berlin, 1975. 
[17] Shimura, G.: Introduction to the arithmetic theory of automorphic functions. Publications of the Mathematical Society of Japan 11. Iwanami Shoten, Tokyo; Princeton University Press, Princeton, N.J., 1971.

[18] Spitzer, F.: Some theorems concerning 2-dimensional Brownian motion. Trans. Amer. Math. Soc. 87 (1958), 187-197.

[19] Thurston, W. P.: Three dimensional manifolds, Kleinian groups and hyperbolic geometry. Bull. Amer. Math. Soc. (N.S.) 6 (1982), no. 3, 357-381.

[20] Watanabe, S.: Asymptotic windings of Brownian motion paths on Riemann surfaces. Acta Appl. Math. 63 (2000), no. 1-3, 441-464.

Recibido: 24 de febrero de 2003

Revisado: 27 de septiembre de 2004

Jacques Franchi

I.R.M.A.

Université Louis Pasteur et CNRS

7 rue René Descartes

67084 Strasbourg cedex. France franchi@math.u-strasbg.fr 